\title{
$O(N)$ Matrix Difference Equations and a Nested Bethe Ansatz
}

\author{
Hrachya M. Babujian*†, Angela Foerster ${ }^{\ddagger}$, and Michael Karowski $₫$ \\ Institut für Theoretische Physik, Freie Universität Berlin, \\ Arnimallee 14, 14195 Berlin, Germany
}

March 14, 2014

\begin{abstract}
A system of $O(N)$-matrix difference equations is solved by means of the offshell version of the nested algebraic Bethe Ansatz. In the nesting process a new object, the $\Pi$-matrix, is introduced to overcome the complexities of the $O(N)$ group structure. The highest weight property of the solutions is proved and some explicit examples are discussed.
\end{abstract}

\section{Introduction}

$O(N)$ Gross-Neveu and $O(N) \sigma$-models are asymptotically free quantum field theories which attract high interest, since they share some common features with QCD. Since perturbation theory fails for these models, exact results, such as exact generalized form factors are desirable and welcome. The concept of a generalized form factor was introduced in [1, 2], where several consistency equations were formulated. Subsequently this approach was developed further and investigated in different models by Smirnov [3]. Generalized form factors are matrix elements of fields with many particle states. To construct these objects explicitly one has to solve generalized Watson's equations which are matrix difference equations. To solve these equations the so called "off-shell Bethe Ansatz" is applied [4, 5, 6]. The conventional Bethe Ansatz introduced by Bethe [7] is used to solve eigenvalue problems and its algebraic formulation was developed by Faddeev and coworkers (see e.g. [8]). The off-shell Bethe Ansatz has been introduced in [9] to solve

\footnotetext{
*Address: Yerevan Physics Institute, Alikhanian Brothers 2, Yerevan, 375036 Armenia

${ }^{\dagger}$ E-mail: babujian@yerphi.am

¥Address: Instituto de Física da UFRGS, Av. Bento Gonçalves 9500, Porto Alegre, RS - Brazil

$\S$ E-mail: angela@if.ufrgs.br

『E-mail: karowski@physik.fu-berlin.de
} 
the Knizhnik-Zamolodchikov equations which are differential equations. In [10] a variant of this technique has been formulated to solve matrix difference equations of the form

$$
K\left(u_{1}, \ldots, u_{i}+\kappa, \ldots, u_{n}\right)=K\left(u_{1} \ldots, u_{i}, \ldots, u_{n}\right) Q\left(u_{1}, \ldots, u_{n}, ; i\right), \quad(i=1, \ldots, n),
$$

where $K(\underline{u})$ is a co-vector valued function, $Q(\underline{u}, i)$ are matrix valued functions and $\kappa$ is a constant to be specified. We use here a co-vector formulation because this is more convenient for the application to the form factor program. For higher rank internal symmetry groups the nested version of this Bethe Ansatz has to be applied. The nested Bethe Ansatz as a method to solve eigenvalue problems was introduced by Yang [11] and further developed by Sutherland [12, 13.

In this article we will solve the $O(N)$ difference equations combining the nested Bethe Ansatz with the off-shell Bethe Ansatz. This procedure is similar to the $S U(N)$ [14] case, where also a nesting procedure is used. However, the algebraic formulation for $O(N)$ is much more intricate because the R-matrix exhibits an extra new term. In addition, for $S U(N)$ we can use the same R-matrix at every level, while for the group $O(N)$ the $\mathrm{R}$-matrix changes after each level. Therefore in our construction a new object, called $\Pi$ matrix, is introduced in order to overcome these difficulties. This provides a systematic formulation of techniques introduced by Tarasov [15] and also used in [16]. In [17] a different procedure was used to solve the $O(N)$ on-shell Bethe Ansatz for even $N$.

The results of this article will be applied in [18] to calculate exact form factors of the $O(N) \sigma-$ and Gross-Neveu models. We should mention that the first computation of form factors for $O(3) \sigma$-model is due to [3] (see also [19, 20]). There are also new developments concerning the connection between 2d Conformal Field Theory (CFT) and integrable models with $N=2$ Super Yang Mills (SYM) theories in different higher dimensions. First, there is a surprising relation between $2 \mathrm{~d}$-conformal blocks and the instanton partition function in $N=2$, 4d-SYM theory [21] (Alday, Gaiotto, Tachikawa - AGT relation) and this is a particular version of the AdS/CFT correspondence which is a more general part of the gauge/string duality. There is also a q-deformation of the AGT relation which connects the $N=25$ d-SYM theory and the q-deformed conformal blocks [22]. This last relation offers new insights and gives the intriguing hope that the form factor program can be used to obtain a deeper understanding of this connection. The solution of the difference equations is the first step to obtain the exact form factors and therefore important physical relations and correlation functions for integrable models. In fact, difference equations play a significant role in various contexts of mathematical physics (see e.g. [23] and references therein).

The article is organized as follows. In Section 2 we recall some results and fix the notation concerning the $O(N)$ R-matrix, the monodromy matrix and some commutation rules. We also introduce a new object, which we call the $\Pi$-matrix and which is a central element in our construction of the nested off-shell Bethe vector. In Section 3 we introduce the nested generalized Bethe Ansatz to solve a system of $O(N)$ difference equations and present the solutions in terms of "Jackson-type Integrals". We introduce a new type of monodromy matrix fulfilling a new type of Yang-Baxter relation and which is adapted to the difference problem. In particular this yields a relatively simple proof of our main 
result, which is Theorem 3.4. In Section 4 we prove the highest weight property of the solutions and calculate the weights. Section 5 contains some examples of solutions of the $O(N)$ difference equations. The appendices provide the more complicated proofs of additional results we have obtained. In particular, in Appendix B we prove that all 7 types of "unwanted terms" in the Bethe ansatz cancel.

\section{General setting and notion of the П-matrix}

\subsection{The $O(N)$ - R-matrix}

Let $V^{1 \ldots n}$ be the tensor product space

$$
V^{1 \ldots n}=V_{1} \otimes \cdots \otimes V_{n},
$$

where the vector spaces $V_{i} \cong \mathbf{C}^{N},(i=1, \ldots, n)$ are copies of the fundamental vector representation space of $O(N)$ with the (real) basis vectors

$$
|\alpha\rangle_{r} \in V, \quad(\alpha=1, \ldots, N) .
$$

It is straightforward to generalize the results of this paper to the case where the $V_{i}$ are vector spaces for other representations. We denote the canonical basis vectors of $V^{1 \ldots n}$ by

$$
\left|\alpha_{1}, \ldots, \alpha_{n}\right\rangle \in V^{1 \ldots n}, \quad\left(\alpha_{i}=1, \ldots, N\right) .
$$

A vector $v^{1 \ldots n} \in V^{1 \ldots n}$ is given in terms of its components by

$$
v^{1 \ldots n}=\sum_{\underline{\alpha}}\left|\alpha_{1}, \ldots, \alpha_{n}\right\rangle_{r} v^{\alpha_{1}, \ldots, \alpha_{n}}
$$

A matrix acting in $V^{1 \ldots n}$ is denoted by

$$
A_{1 \ldots n}: V^{1 \ldots n} \rightarrow V^{1 \ldots n} .
$$

We will also use the dual space $V_{1 \ldots n}=\left(V^{1 \ldots n}\right)^{\dagger}$.

The $O(N)$ spectral parameter dependent R-matrix was found by ZamolodchikovZamolodchikov [24] $]^{1}$. It acts on the tensor product of two (fundamental) representation spaces of $O(N)$. It may be written as

$$
R_{12}\left(u_{12}\right)=\left(\mathbf{1}_{12}+c\left(u_{12}\right) \mathbf{P}_{12}+d\left(u_{12}\right) \mathbf{K}_{12}\right): V^{12} \rightarrow V^{21},
$$

where $\mathbf{P}_{12}$ is the permutation operator, $\mathbf{K}_{12}$ the annihilation-creation operator and $u_{12}=$ $u_{1}-u_{2}$. Here and in the following we associate to each space $V_{i}$ a variable (spectral parameter) $u_{i} \in \mathbf{C}$. The components of the R-matrix are

$$
R_{\alpha \beta}^{\delta \gamma}\left(u_{12}\right)=\delta_{\alpha}^{\gamma} \delta_{\beta}^{\delta}+\delta_{\alpha}^{\delta} \delta_{\beta}^{\gamma} c\left(u_{12}\right)+\delta^{\gamma \delta} \delta_{\alpha \beta} d\left(u_{12}\right)
$$

\footnotetext{
${ }^{1}$ We use here the normalization $R=S / \sigma_{2}$ and the parameterization $u=\theta / i \pi \nu$ which is more convenient for our purpose.
} 
from which $\mathbf{P}_{12}$ and $\mathbf{K}_{12}$ can be read off. The functions

$$
c(u)=\frac{-1}{u}, \quad d(u)=\frac{1}{u-1 / \nu}, \quad \nu=\frac{2}{N-2}
$$

are obtained as the rational solution of the Yang-Baxter equation

$$
R_{12}\left(u_{12}\right) R_{13}\left(u_{13}\right) R_{23}\left(u_{23}\right)=R_{23}\left(u_{23}\right) R_{13}\left(u_{13}\right) R_{12}\left(u_{12}\right),
$$

where we have employed the usual notation [11]. We will also use

$$
\tilde{R}(u)=R(u) / a(u)
$$

with

$$
a(u)=1+c(u)=\frac{u-1}{u} .
$$

The "unitarity" of the R-matrix reads as

$$
\tilde{R}_{21}\left(u_{21}\right) \tilde{R}_{12}\left(u_{12}\right)=1
$$

and the three eigenvalues of the R-matrix are

$$
R_{ \pm}(u)=1 \pm c(u)=\frac{u \mp 1}{u}, R_{0}=1+c(u)+N d(u) .
$$

The crossing relation may be written as

$$
R_{12}\left(u_{12}\right)=\mathbf{C}^{2 \overline{2}} R_{\overline{2} 1}\left(\hat{u}_{12}\right) \mathbf{C}_{\overline{2} 2}=\mathbf{C}^{1 \overline{1}} R_{2 \overline{1}}\left(\hat{u}_{12}\right) \mathbf{C}^{\overline{1} 1}
$$

where $\hat{u}=1 / \nu-u$. Here $\mathbf{C}^{1 \overline{1}}$ and $\mathbf{C}_{1 \overline{1}}$ are the charge conjugation matrices. Their matrix elements are $\mathbf{C}^{\alpha \bar{\beta}}=\mathbf{C}_{\alpha \bar{\beta}}=\delta_{\alpha \beta}$ where $\bar{\beta}$ denotes the anti-particle of $\beta$. In the real basis used up to now the particles are chargeless which means that $\bar{\beta}=\beta$ and $\mathbf{C}$ is diagonal.

In the following we will use instead of the real basis $|\alpha\rangle_{r},(\alpha=1,2, \ldots, N)$ the complex basis given by

$$
\begin{aligned}
& |\alpha\rangle=\frac{1}{\sqrt{2}}\left(|2 \alpha-1\rangle_{r}+i|2 \alpha\rangle_{r}\right) \\
& |\bar{\alpha}\rangle=\frac{1}{\sqrt{2}}\left(|2 \alpha-1\rangle_{r}-i|2 \alpha\rangle_{r}\right)
\end{aligned}
$$

for $\alpha=1,2, \ldots,[N / 2]$. If $N$ is odd there is in addition $|0\rangle=|\overline{0}\rangle=|N\rangle_{r}$. The weight vector $w=\left(w_{1}, \ldots, w_{[N / 2]}\right)$ and the charges of the one-particle states are given by

$$
\begin{aligned}
& \text { for }|\alpha\rangle: w_{k}=\delta_{k \alpha} \quad, \quad Q=1 \\
& \text { for }|\bar{\alpha}\rangle: w_{k}=-\delta_{k \alpha} \quad, \quad Q=-1 \\
& \text { for }|0\rangle: w_{k}=0 \quad, Q=0 \text {. }
\end{aligned}
$$


Remark 2.1 For even $N$ this means that we consider $O(N)$ as a subgroup of $U(N / 2)$ and the charge $Q$ is its $U(1)$ charge. For $N=3$ we may identify the particles $1, \overline{1}, 0$ with the pions $\pi_{ \pm}, \pi_{0}$.

The highest weight eigenvalue of the R-matrix is

$$
R_{11}^{11}(u)=R_{+}(u)=a(u) .
$$

We order the states as: $1,2, \ldots,(0), \ldots, \overline{2}, \overline{1}$ ( 0 only for $N$ odd). Then the charge conjugation matrix in the complex basis is of the form

$$
\begin{aligned}
\mathbf{C}^{\delta \gamma} & =\delta^{\delta \bar{\gamma}}, \mathbf{C}_{\alpha \beta}=\delta_{\alpha \bar{\beta}} \\
\mathbf{C} & =\left(\begin{array}{ccccc}
0 & \cdots & 0 & \cdots & 1 \\
\vdots & \ddots & \vdots & \cdot & \vdots \\
0 & \cdots & 1 & \cdots & 0 \\
\vdots & \cdot & \vdots & \ddots & \vdots \\
1 & \cdots & 0 & \cdots & 0
\end{array}\right) .
\end{aligned}
$$

The annihilation-creation matrix in (5) may be written as

$$
\mathbf{K}_{\alpha \beta}^{\delta \gamma}=\mathbf{C}^{\delta \gamma} \mathbf{C}_{\alpha \beta} \text {. }
$$

\subsection{The monodromy matrix}

We consider a state with $n$ particles and as is usual in the context of the algebraic Bethe Ansatz we define [25, 8] the monodromy matrix by

$$
T_{1 \ldots n, 0}\left(\underline{u}, u_{0}\right)=R_{10}\left(u_{10}\right) \cdots R_{n 0}\left(u_{n 0}\right)
$$

with $\underline{u}=u_{1}, \ldots, u_{n}$. It is a matrix acting in the tensor product of the "quantum space" $V^{1 \ldots n}=V_{1} \otimes \cdots \otimes V_{n}$ and the "auxiliary space" $V_{0}$. All vector spaces $V_{i}$ are isomorphic to a space $V$ whose basis vectors label all kinds of particles. Here $V \cong \mathbb{C}^{N}$ is the space of the vector representation of $O(N)$.

Suppressing the indices $1 \ldots n$ we write the monodromy matrix as (following the notation of Tarasov [15])

$$
T_{\alpha}^{\alpha^{\prime}}=\left(\begin{array}{ccc}
A_{1} & \left(B_{1}\right)_{\dot{\alpha}} & B_{2} \\
\left(C_{1}\right)^{\alpha^{\prime}} & \left(A_{2}\right)_{\dot{\alpha}}^{\alpha^{\prime}} & \left(B_{3}\right)^{\dot{\alpha}^{\prime}} \\
C_{2} & \left(C_{3}\right)_{\dot{\alpha}} & A_{3}
\end{array}\right)
$$

where $\alpha, \alpha^{\prime}$ assume the values $1,2, \ldots,(0), \ldots, \overline{2}, \overline{1}$ corresponding to the basis vectors of the auxiliary space $V \cong \mathbb{C}^{N}$ and $\stackrel{\circ}{\alpha}, \stackrel{\circ}{\alpha}^{\prime}$ assume the values $2, \ldots,(0), \ldots, \overline{2}$ corresponding to the basis vectors of $\stackrel{\circ}{V} \cong \mathbb{C}^{N-2}$. We will also use the notation $A=A_{1}, B=B_{1}, C=C_{1}$ and $D=A_{2}$ which is an $(N-2) \times(N-2)$ matrix in the auxiliary space. The Yang-Baxter algebra relation for the $\mathrm{R}$-matrix (8) yields

$$
T_{1 \ldots n, a}\left(\underline{u}, u_{a}\right) T_{1 \ldots n, b}\left(\underline{u}, u_{b}\right) R_{a b}\left(u_{a b}\right)=R_{a b}\left(u_{a b}\right) T_{1 \ldots n, b}\left(\underline{u}, u_{b}\right) T_{1 \ldots n, a}\left(\underline{u}, u_{a}\right) .
$$




\section{$2.3 \quad$ A lemma}

In our approach of the algebraic Bethe Ansatz the following lemma replaces commutation rules of the entries of the monodromy matrix. In the conventional approach one derives them from the Yang-Baxter algebra relations (14) and uses them for the algebraic Bethe Ansatz.

Lemma 2.2 For the monodromy matrix the following identity holds

$$
\begin{aligned}
T_{1 \ldots n, a}(\underline{u}, v)=\mathbf{1}_{1} \ldots \mathbf{1}_{n} \mathbf{1}_{a}+\sum_{i=1}^{n} c\left(u_{i}-v\right) & R_{1 a}\left(u_{1 i}\right) \ldots \mathbf{P}_{i a} \ldots R_{n a}\left(u_{n i}\right) \\
& +\sum_{j=1}^{n} d\left(u_{i}-v\right) R_{1 a}\left(\hat{u}_{i 1}\right) \ldots \mathbf{K}_{i a} \ldots R_{n a}\left(\hat{u}_{i n}\right)
\end{aligned}
$$

with $\hat{u}=1 / \nu-u$.

Proof. The R-matrix $R(u)$ (see (6) and (7)) is meromorphic and has simple poles at $u=0$ and $u=1 / \nu$ due to the form of $c(u)$ and $d(u)$ such that

$$
\begin{aligned}
\operatorname{Res}_{v=u_{i}} T_{1 \ldots n, a}(\underline{u}, v) & =\operatorname{Res}_{v=u_{i}} c\left(u_{i}-v\right) R_{1 a}\left(u_{1 i}\right) \ldots \mathbf{P}_{i a} \ldots R_{n a}\left(u_{n i}\right) \\
\operatorname{Res}_{v=u_{i}-1 / \nu} T_{1 \ldots n, a}(\underline{u}, v) & =\operatorname{Res}_{v=u_{i}-1 / \nu} d\left(u_{j}-v\right) R_{1 a}\left(\hat{u}_{i 1}\right) \ldots \mathbf{K}_{i a} \ldots R_{m a}\left(\hat{u}_{i n}\right)
\end{aligned}
$$

holds. The claim follows by Liouville's theorem because $\lim _{v \rightarrow \infty} T_{1 \ldots n, a}(\underline{u}, v)=\mathbf{1}_{1} \ldots \mathbf{1}_{n} \mathbf{1}_{a}$.

Similarly we have for the crossed monodromy matrix

$$
T_{a, 1 \ldots n}(v, \underline{u})=R_{a n}\left(v-u_{n}\right) \ldots R_{a 1}\left(v-u_{1}\right)
$$

the relation

$$
\begin{aligned}
T_{a, 1 \ldots n}(v, \underline{u})=\mathbf{1}_{a} \mathbf{1}_{1} \ldots \mathbf{1}_{n}+\sum_{i=1}^{n} c(v & \left.-u_{i}\right) R_{a n}\left(u_{i n}\right) \ldots \mathbf{P}_{a i} \ldots R_{a 1}\left(u_{i 1}\right) \\
& +\sum_{i=1}^{n} d\left(v-u_{i}\right) R_{a m}\left(\hat{u}_{m i}\right) \ldots \mathbf{K}_{a i} \ldots R_{a 1}\left(\hat{u}_{1 i}\right) .
\end{aligned}
$$

Note that the crossing relation 100 implies

$$
T_{a, 1 \ldots n}\left(v_{a}, \underline{u}\right)=\mathbf{C}_{b a} T_{1 \ldots n, b}\left(\underline{u}, v_{b}\right) \mathbf{C}^{a b}
$$

with $v_{b}=v_{a}-1 / \nu$. 


\subsection{The Matrix $\Pi$}

The nested Bethe Ansatz relies on the principle that after each level the rank of the group (or quantum group) is reduced by one. For $S U(N)$ the rank is $N-1$ and for $O(N)$ it is $[N / 2]$. This means that the dimension of the vector representation (where the R-matrix usually acts) is reduced by 1 for the case of $S U(N)$ and by 2 for case of $O(N)$. A more essential difference is that for $S U(N)$ one can use in every level the same R-matrix, because (with a suitable normalization and parameterization) the $S U(N)$ R-matrix does not depend on $N$. In contrast for $O(N)$ the R-matrix changes after each level, because it depends on $N$. Therefore we need a new object called matrix $\Pi$, which maps the $O(N)$ R-matrix to the $O(N-2)$ one. We use the notation

$$
\begin{aligned}
& \stackrel{\circ}{R}(u)=R(u, N-2)=\mathbf{1}+\mathbf{P} c(u)+\mathbf{K} \stackrel{\circ}{d}(u) \\
& \stackrel{\circ}{d}(u)=\frac{1}{u-1 / \stackrel{\circ}{\nu}}
\end{aligned}
$$

with $\stackrel{\circ}{\nu}=2 /(N-4)$. The components of the R-matrix $\stackrel{\circ}{R}(u)$ will be denoted by

$$
\stackrel{\circ}{R}_{\stackrel{\circ}{\delta} \stackrel{\gamma}{\alpha}}^{\alpha}(u), \stackrel{\circ}{\alpha}, \stackrel{\circ}{\beta}, \stackrel{\circ}{\gamma}, \stackrel{\circ}{\delta}=2,3, \ldots,(0), \ldots, \overline{3}, \overline{2} .
$$

In addition to $V^{1 \ldots m}=V_{1} \otimes \cdots \otimes V_{m}(1)$ we introduce

$$
\stackrel{\circ}{V}^{1 \ldots m}=\stackrel{\circ}{V}_{1} \otimes \cdots \otimes \stackrel{\circ}{V}_{m}
$$

where the vector spaces $\stackrel{\circ}{V}_{i} \cong \mathbf{C}^{N-2},(i=1, \ldots, m)$ are considered as fundamental (vector) representation spaces of $O(N-2)$. The space $V_{i}$ is spanned by the complex basis vectors $|1\rangle,|2\rangle, \ldots,|\overline{2}\rangle,|\overline{1}\rangle$ and $\stackrel{\circ}{V}_{i}$ by $|2\rangle, \ldots,|\overline{2}\rangle$.

Definition 2.3 We define the map

$$
\Pi_{1 \ldots m}: V^{1 \ldots m} \rightarrow \stackrel{\circ}{V}^{1 \ldots m}
$$

recursively by $\Pi_{1}=\pi_{1}$ and

$$
\Pi_{1 \ldots m}(\underline{u})=\left(\pi_{1} \Pi_{2 \ldots m}\right) \bar{e}_{a} T_{1 \ldots m, a}\left(\underline{u}, u_{a}\right) \bar{e}^{a}
$$

with the projector $\pi: V \rightarrow \stackrel{\circ}{V} \subset V$, the monodromy matrix 112) and $u_{a}=u_{1}-1$. The vector $\bar{e}^{a} \in V_{a}$ (acting in the auxiliary space of $T_{1 \ldots m, a}$ ) and the co-vector $\bar{e}_{a} \in\left(V_{a}\right)^{\dagger}$ correspond to the state $\overline{1}$ and have the components $\bar{e}^{\alpha}=\delta_{\overline{1}}^{\alpha}, \bar{e}_{\alpha}=\delta_{\alpha}^{\overline{1}}$.

Lemma 2.4 In particular for $m=2$ the matrix $\Pi_{12}\left(u_{1}, u_{2}\right)$ may be written as

$$
\Pi_{12}(\underline{u})=\pi_{1} \pi_{2}+f\left(u_{12}\right) \stackrel{\circ}{\mathbf{C}}^{12} \bar{e}_{1} e_{2}
$$

with $e_{2}=\mathbf{C}_{2 a} \bar{e}^{a}$ and $f(u)=-d(1-u)$. It satisfies the fundamental relation

$$
\stackrel{\circ}{R}_{12}\left(u_{12}\right) \Pi_{12}\left(u_{1}, u_{2}\right)=\Pi_{21}\left(u_{2}, u_{1}\right) R_{12}\left(u_{12}\right),
$$

where $\stackrel{\circ}{R}_{12}$ is the $O(N-2) R$-matrix. 
Proof. Equation (21) can be easily derived

$$
\begin{aligned}
\Pi_{12}(\underline{u}) & =\pi_{1} \pi_{2} \bar{e}_{a} T_{12, a}\left(\underline{u}, u_{a}\right) \bar{e}^{a} \\
& =\pi_{1} \pi_{2} \bar{e}_{a} R_{1 a}\left(u_{1}-u_{a}\right) R_{2 a}\left(u_{2}-u_{a}\right) \bar{e}^{a} \\
& =\pi_{1} \pi_{2} \bar{e}_{a}\left(\mathbf{1}_{1} \mathbf{1}_{a}+c(1) \mathbf{P}_{1 a}\right)\left(\mathbf{1}_{2} \mathbf{1}_{a}+d\left(u_{21}+1\right) \mathbf{K}_{2 a}\right) \bar{e}^{a} \\
& =\pi_{1} \pi_{2}+c(1) d\left(u_{21}+1\right) \stackrel{\mathbf{C}}{12}^{12} \bar{e}_{1} e_{2} .
\end{aligned}
$$

Use has been made of $c(1)=-1$ and $\pi_{1} \pi_{2} \bar{e}_{a} \mathbf{P}_{1 a} \mathbf{K}_{2 a} \bar{e}^{a}=\stackrel{\circ}{\mathbf{C}}^{12} \bar{e}_{1} e_{2}$. Equation 22$]$ is derived for all components. Obviously

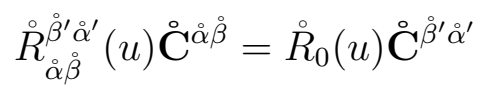

holds, where the scalar R-matrix eigenvalue is (see (9))

$$
\stackrel{\circ}{R}_{0}(u)=a(u)+(N-2) \stackrel{\circ}{d}(u) .
$$

Therefore the relations

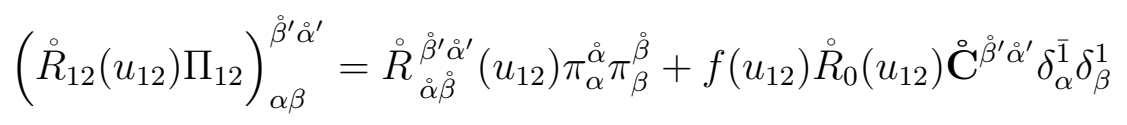

and

$$
\left(\Pi_{21} R_{12}\left(u_{12}\right)\right)_{\alpha \beta}^{{\stackrel{\beta^{\prime}}{\alpha} \alpha^{\prime}}^{\prime}}=\pi_{\beta^{\prime}}^{\hat{\beta}^{\prime}} \pi_{\alpha^{\prime}}^{\alpha^{\prime}} R_{\alpha \beta}^{\beta^{\prime} \alpha^{\prime}}\left(u_{12}\right)+f\left(u_{21}\right){\stackrel{\circ}{\mathbf{C}^{\prime} \alpha^{\prime} \alpha^{\prime}}}^{\overline{1} 1} R_{\alpha \beta}\left(u_{12}\right)
$$

are valid. The claim of the lemma is then equivalent to

$$
\begin{array}{ll}
(i): \stackrel{\circ}{d}(u)=d(u)+f(-u) d(u) & \text { for } \alpha \text { or } \beta \neq 1, \overline{1} \\
(i i): 0=d(u)+f(-u)(1+d(u)) & \text { for } \alpha=1, \quad \beta=\overline{1} \\
(i i i): f(u) \stackrel{\circ}{R}_{0}(u)=d(u)+f(-u)(c(u)+d(u)) & \text { for } \alpha=\overline{1}, \quad \beta=1 .
\end{array}
$$

These equations may be easily checked with the amplitudes (7).

These results can be extended to general $m$, as presented below.

Lemma 2.5 The matrix $\Pi_{1 \ldots m}(\underline{u})$ satisfies

(a) in addition to (20) the recursion relation

$$
\Pi_{1 \ldots m}(\underline{u})=\left(\Pi_{1 \ldots m-1} \pi_{m}\right) \bar{e}_{b} T_{1 \ldots m, b}\left(\underline{u}, u_{b}\right) \bar{e}^{b}
$$

with $u_{b}=u_{m}-1 / \nu+1$, and

(b) the fundamental relation

$$
\stackrel{\circ}{R}_{i j}\left(u_{i j}\right) \Pi_{\ldots i j \ldots}(\underline{u})=\Pi_{\ldots j i \ldots}(\underline{u}) R_{i j}\left(u_{i j}\right) .
$$

(c) The matrix $\bar{e}_{0} T_{1 \ldots m, 0}\left(\underline{u}, u_{0}\right) \bar{e}^{0}$ acts on $\Pi_{1 \ldots m}(\underline{u})$ as the unit matrix for arbitrary $u_{0}$

$$
\Pi_{1 \ldots m}(\underline{u}) \bar{e}_{0} T_{1 \ldots m, 0}\left(\underline{u}, u_{0}\right) \bar{e}^{0}=\Pi_{1 \ldots m}(\underline{u}) .
$$


(d) Special components of $\Pi$ satisfy

$$
\begin{aligned}
& \Pi_{1 \alpha_{2} \ldots \alpha_{m}}^{\alpha_{1} \ldots \alpha_{m}}\left(u_{1}, \ldots, u_{m}\right)=0 \\
& \Pi_{\alpha \alpha_{2} \ldots \alpha_{m}}^{\alpha_{1} \ldots \dot{\alpha}_{m}}\left(u_{1}, \ldots, u_{m}\right)=\delta_{\alpha}^{\alpha_{\alpha}} \prod_{\alpha_{2} \ldots \alpha_{m}}^{\alpha_{2} \ldots \alpha_{m}}\left(u_{2}, \ldots, u_{m}\right) \\
& \Pi_{\alpha_{1} \ldots \alpha_{m-1} \overline{1}}^{\alpha_{1} \ldots \stackrel{\circ}{m}_{m}}\left(u_{1}, \ldots, u_{m}\right)=0 \\
& \prod_{\alpha_{1} \ldots \alpha_{m-1} \dot{\alpha}}^{\dot{\alpha}_{1} \ldots \alpha_{m}}\left(u_{1}, \ldots, u_{m}\right)=\prod_{\alpha_{1} \ldots \alpha_{m-1}}^{\alpha_{1} \ldots \alpha_{m-1}}\left(u_{1}, \ldots, u_{m-1}\right) \delta_{\dot{\alpha}}^{\alpha_{m}} .
\end{aligned}
$$

with $\stackrel{\circ}{\alpha} \neq 1, \overline{1}$.

The proof of this Lemma is presented in appendix A.

The definitions (20) and (23) can be rewritten as (see also lemma 2.4 for $m=2$ )

$$
\begin{aligned}
\Pi_{1 \ldots m}(\underline{u}) & =\pi_{1} \Pi_{2 \ldots m}+\sum_{j=2}^{m} f\left(u_{1 j}\right) \stackrel{\circ}{R}_{j j-1} \cdots \stackrel{\circ}{R}_{j 2} \stackrel{\circ}{ }^{1 j} \Pi_{2 \ldots . . . . m} \bar{e}_{1} e_{j} R_{j m} \cdots R_{j j+1} \\
& =\Pi_{1 \ldots m-1} \pi_{m}+\sum_{j=1}^{m-1} f\left(u_{j m}\right) \stackrel{\circ}{R}_{j+1 j} \cdots \stackrel{\circ}{R}_{m-1 j} \stackrel{\circ}{\mathbf{C}}^{j m} \Pi_{1 \ldots \hat{\jmath} \ldots m-1} \bar{e}_{j} R_{1 j} \cdots R_{j-1 j} e_{m} .
\end{aligned}
$$

\section{The $O(N)$ - difference equation}

Let $K_{1 \ldots n}(\underline{u}) \in V_{1 \ldots n}$ be a co-vector valued function of $\underline{u}=u_{1}, \ldots, u_{n}$ with values in $V_{1 \ldots n}$. The components of this vector are denoted by

$$
K_{\alpha_{1} \ldots \alpha_{n}}(\underline{u}), \quad\left(\alpha_{i}=1,2, \ldots,(0), \ldots, \overline{2}, \overline{1}\right) .
$$

The following symmetry and periodicity properties of this function are supposed to be valid:

\section{Conditions 3.1}

(i) The symmetry property under the exchange of two neighboring spaces $V_{i}$ and $V_{j}$ and the variables $u_{i}$ and $u_{j}$, at the same time, is given by

$$
K_{\ldots i j \ldots}\left(\ldots, u_{i}, u_{j}, \ldots\right)=K_{\ldots j i \ldots}\left(\ldots, u_{j}, u_{i}, \ldots\right) \tilde{R}_{i j}\left(u_{i j}\right),
$$

where $\tilde{R}(u)=R(u) / a(u)$ and $R(u)$ is the $O(N) R$-matrix.

(ii) The system of matrix difference equations holds

$$
K_{1 \ldots n}\left(\ldots, u_{i}^{\prime}, \ldots\right)=K_{1 \ldots n}\left(\ldots, u_{i}, \ldots\right) Q_{1 \ldots n}(\underline{u} ; i), \quad(i=1, \ldots, n)
$$

with $u_{i}^{\prime}=u_{i}+2 / \nu$. The matrix $Q_{1 \ldots n}(\underline{u} ; i) \in \operatorname{End}\left(V^{1 \ldots n}\right)$ is defined as the trace

$$
Q_{1 \ldots n}(\underline{u} ; i)=\operatorname{tr}_{0} \tilde{T}_{Q, 1 \ldots n, 0}(\underline{u}, i)
$$

of a modified monodromy matrix

$$
\tilde{T}_{Q, 1 \ldots n, 0}(\underline{u}, i)=\tilde{R}_{10}\left(u_{1}-u_{i}^{\prime}\right) \cdots \mathbf{P}_{i 0} \cdots \tilde{R}_{n 0}\left(u_{n}-u_{i}\right) .
$$


The Yang-Baxter equations for the R-matrix guarantee that these properties are compatible. The shift of $2 / \nu$ in eq. (33) could be replaced by an arbitrary $\kappa$. For the application to the form factor problem, however, it is fixed to be equal to $2 / \nu$ in order to be compatible with crossing symmetry. Instead of the Yang-Baxter relation (14) the modified monodromy matrix $\tilde{T}_{Q}$ satisfies the Zapletal rules [14, 4]. We have for $i=1, \ldots, n$

$$
\tilde{T}_{Q}(\underline{u} ; i) T_{0}\left(\underline{u}^{\prime}, v\right) R_{i 0}\left(u_{i}-v\right)=R_{i 0}\left(u_{i}^{\prime}-v\right) T_{0}(\underline{u}, v) \tilde{T}_{Q}(\underline{u} ; i)
$$

with $\underline{u}^{\prime}=u_{1}, \ldots, u_{i}^{\prime}, \ldots, u_{n}$ and $u_{i}^{\prime}=u_{i}+2 / \nu$. The $Q_{1 \ldots n}(\underline{u} ; i)$ satisfy the commutation rules

$$
\begin{aligned}
Q_{1 \ldots n}\left(\ldots u_{i} \ldots u_{j} \ldots ; i\right) Q_{1 \ldots n}\left(\ldots u_{i}^{\prime} \ldots u_{j} \ldots ; j\right) \\
\quad=Q_{1 \ldots n}\left(\ldots u_{i} \ldots u_{j} \ldots ; j\right) Q_{1 \ldots n}\left(\ldots u_{i} \ldots u_{j}^{\prime} \ldots ; i\right) .
\end{aligned}
$$

The following Proposition is obvious

Proposition 3.2 Let the vector valued function $K_{1 \ldots n}(\underline{u}) \in V_{1 \ldots n}$ satisfy $(i)$. Then for all $i=1, \ldots, n$ the relations (3.2) are equivalent to each other and also equivalent to the following periodicity property under cyclic permutation of the spaces and the variables

$$
K_{\alpha_{1} \alpha_{2} \ldots \alpha_{n}}\left(u_{1}^{\prime}, u_{2}, \ldots, u_{n}\right)=K_{\alpha_{2} \ldots \alpha_{n} \alpha_{1}}\left(u_{2}, \ldots, u_{n}, u_{1}\right) .
$$

Remark 3.3 The equations 32. 37) imply Watson's equations for the form factors [26].

Because of proposition 3.2 we mainly consider $Q_{1 \ldots n}(\underline{u}, i)$ for $i=1$

$$
Q_{1 \ldots n}(\underline{u})=\operatorname{tr}_{0} \tilde{T}_{Q, 1 \ldots n, 0}(\underline{u})=\prod_{k=2}^{n} \frac{1}{a\left(v_{k i}\right)} \operatorname{tr}_{0} T_{Q, 1 \ldots n, 0}(\underline{u})
$$

with $T_{Q, 1 \ldots n, 0}(\underline{u})=T_{Q, 1 \ldots n, 0}(\underline{u}, 1)$. In analogy to eq. (13) we introduce (suppressing the indices $1 \ldots n$ )

$$
T_{Q}(\underline{u}) \equiv\left(\begin{array}{ccc}
A_{Q}(\underline{u}) & B_{Q}(\underline{u}) & B_{Q, 2}(\underline{u}) \\
C_{Q}(\underline{u}) & D_{Q}(\underline{u}) & B_{Q, 3}(\underline{u}) \\
C_{Q, 2}(\underline{u}) & C_{Q, 3}(\underline{u}) & A_{Q, 3}(\underline{u})
\end{array}\right) .
$$

\subsection{The off-shell Bethe Ansatz}

We will express the co-vector valued function $K_{\underline{\alpha}}(\underline{u})$ in terms of the co-vectors

$$
\Psi_{\underline{\alpha}}(\underline{u}, \underline{v})=L_{\underline{\underline{\beta}}}(\underline{v}) \Phi_{\underline{\alpha}}^{\stackrel{\leftrightarrow}{\beta}}(\underline{u}, \underline{v})=(L(\underline{v}) \Phi(\underline{u}, \underline{v}))_{\underline{\alpha}},
$$

where summation over $\stackrel{\circ}{\beta}_{1}, \ldots, \stackrel{\circ}{\beta}_{m}, \stackrel{\circ}{\beta}_{i}=2, \ldots, 0, \ldots, \overline{2}$ is assumed and $L_{\dot{\beta}}(\underline{v})$ is a co-vector valued function with values in ${\stackrel{\circ}{V_{1} \ldots m}} \simeq \mathbb{C}^{N-2} \otimes \cdots \otimes \mathbb{C}^{N-2}$. We assume that for $L_{\dot{\beta}}(\underline{v})$ the 
higher level conditions of 3.1 hold with $R$ and $Q$ replaced by $\stackrel{\circ}{R}$ and $\stackrel{\circ}{Q}$ (which means $N$ is replaced by $N-2$ )

$$
\begin{array}{ll}
(\mathrm{i})^{(1)}: & L_{\ldots i j \ldots}\left(\ldots, v_{i}, v_{j}, \ldots\right)=L_{\ldots j i \ldots}\left(\ldots, v_{j}, v_{i}, \ldots\right) \tilde{\stackrel{̊}{R}}_{i j}\left(v_{i j}\right) \\
(\mathrm{ii})^{(1)}: & L_{1 \ldots m}\left(\ldots, v_{i}^{\prime}, \ldots\right)=L_{1 \ldots n}\left(\ldots, v_{i}, \ldots\right) \stackrel{Q}{Q}_{1 \ldots m}(\underline{v}, i) .
\end{array}
$$

The Bethe Ansatz states are 2

$$
\Phi_{\underline{\alpha}}^{\underline{\beta}}(\underline{u}, \underline{v})=\Pi_{\underline{\beta}}^{\underline{\beta}}(\underline{v})\left(\Omega T_{1}^{\beta_{m}}\left(\underline{u}, v_{m}\right) \ldots T_{1}^{\beta_{1}}\left(\underline{u}, v_{1}\right)\right)_{\underline{\alpha}} .
$$

The reference state $\Omega$ ("pseudo-vacuum") is the highest weight co-vector (with weights $w=(n, 0, \ldots, 0))$

$$
\Omega_{\underline{\alpha}}=\delta_{\alpha_{1}}^{1} \cdots \delta_{\alpha_{n}}^{1}
$$

It satisfies

$$
\begin{gathered}
\Omega T(\underline{u}, v)=\Omega\left(\begin{array}{ccc}
a_{1}(\underline{u}, v) & 0 & 0 \\
* & a_{2}(\underline{u}, v) & 0 \\
* & * & a_{3}(\underline{u}, v)
\end{array}\right), \\
a_{1}(\underline{u}, v)=\prod_{k=1}^{n} a\left(u_{k}-v\right), a_{2}(\underline{u}, v)=1, a_{3}(\underline{u}, v)=\prod_{k=1}^{n}\left(1+d\left(u_{k}-v\right)\right) .
\end{gathered}
$$

We also have for $T_{Q}(\underline{u})=T_{Q}(\underline{u}, 1)$

$$
\Omega T_{Q}(\underline{u})=\Omega \prod_{k=2}^{n} a\left(u_{k 1}\right)\left(\begin{array}{ccc}
1 & 0 & 0 \\
* & 0 & 0 \\
* & 0 & 0
\end{array}\right) .
$$

The system of difference equations (33) can be solved by means of a nested "off-shell" Bethe Ansatz. The first level is given by the

\section{BETHE ANSATZ 1}

$$
K_{\underline{\alpha}}(\underline{u})=\sum_{\underline{v}} g(\underline{u}, \underline{v}) \Psi_{\underline{\alpha}}(\underline{u}, \underline{v})
$$

where $\Psi$ is given by 40 and

$$
g(\underline{u}, \underline{v})=\prod_{i=1}^{n} \prod_{j=1}^{m} \psi\left(u_{i}-v_{j}\right) \prod_{1 \leq i<j \leq m} \tau\left(v_{i}-v_{j}\right) .
$$

${ }^{2}$ The $\Phi_{\underline{\alpha}}^{\underline{\beta}}$ are generalizations of the states introduced by Tarasov in [15]. 
The functions $\psi(u)$ and $\tau(v)$ satisfy the functional equations

$$
\psi\left(u^{\prime}\right)=a(u) \psi(u), \tau\left(v^{\prime}\right) a\left(v^{\prime}\right)=a(-v) \tau(v) .
$$

with $u^{\prime}=u+2 / \nu$ The summation over $\underline{v}$ is specified by

$$
\underline{v}=\left(v_{1}, \ldots, v_{m}\right)=\left(\tilde{v}_{1}-2 l_{1} / \nu, \ldots, \tilde{v}_{m}-2 l_{m} / \nu\right), l_{i} \in \mathbf{Z},
$$

where the $\tilde{v}_{i}$ are arbitrary constants.

The sums (47) are also called "Jackson-type Integrals" (see e.g. [10] and references therein). Solutions of (49) are

$$
\begin{aligned}
\psi(u) & =\frac{\Gamma\left(-\frac{1}{2} \nu+\frac{u}{2} \nu\right)}{\Gamma\left(\frac{u}{2} \nu\right)} \\
\tau(v) & =v \frac{\Gamma\left(\frac{1}{2} \nu+\frac{v}{2} \nu\right)}{\Gamma\left(1-\frac{1}{2} \nu+\frac{v}{2} \nu\right)}
\end{aligned}
$$

We are now in a position to formulate the main result of this paper.

Theorem 3.4 Let the co-vector valued function $K_{1 \ldots n}(\underline{u}) \in V_{1 \ldots n}$ be given by the Bethe Ansatz 1 and let $g(\underline{x}, \underline{u})$ be of the form (48). If in addition the co-vector valued function $L_{1 \ldots m}(\underline{v}) \in \stackrel{\circ}{V}_{1 \ldots m}$ satisfies the properties $(i)^{(1)}$ and $(i i)^{(1)}$, i.e. equations (32) and (33) for $O(N-2)$, then $K_{1 \ldots n}(\underline{u})$ satisfies the equations (32) and (33) for $O(N)$, i.e. $K_{1 \ldots n}(\underline{u})$ is a solution of the set of difference equations.

The proof of this theorem can be found in appendix B.

Iterating (47), (40) and theorem 3.4 we obtain the nested off-shell Bethe Ansatz with levels $k=1, \ldots,[(N-1) / 2]$. The Ansatz for level $k$ reads

$$
\begin{aligned}
K_{1 \ldots n_{k-1}}^{(k-1)}\left(\underline{u}^{(k-1)}\right) & =\sum_{\underline{u}^{(k)}} g^{(k-1)}\left(\underline{u}^{(k-1)}, \underline{u}^{(k)}\right) \Psi_{1 \ldots n_{k-1}}^{(k-1)}\left(\underline{u}^{(k-1)}, \underline{u}^{(k)}\right) \\
\Psi_{1 \ldots n_{k-1}}^{(k-1)}\left(\underline{u}^{(k-1)}, \underline{u}^{(k)}\right) & =\left(K^{(k)}\left(\underline{u}^{(k)}\right) \Phi^{(k-1)}\left(\underline{u}^{(k-1)}, \underline{u}^{(k)}\right)\right)_{1 \ldots n_{k-1}},
\end{aligned}
$$

where $\Phi^{(k)}$ is the Bethe Ansatz state (43) and $g^{(k)}$ the function 48 for $O(N-2 k)$. The start of the iteration is given by a $1 \leq k_{\max }<[(N+1) / 2]$ with

$$
\Psi_{\alpha_{1} \ldots \alpha_{n_{k-1}}}^{\left(k_{\max }-1\right)}=\delta_{\alpha_{1}}^{k_{\max }} \ldots \delta_{\alpha_{n_{k-1}}}^{k_{\max }}, \text { and } n_{k}=0 \text { for } k \geq k_{\max }
$$

which is the reference state of level $k_{\max }-1$ and trivially fulfills the Conditions 3.1 . The start of the iteration for $k_{\max }=[(N+1) / 2]$ is given by the solution for $O(3)$ for $N$ odd and $O(4)$ for $N$ even. These two case are investigated below.

Corollary 3.5 The system of $O(N)$ matrix difference equations (33) is solved by the nested Bethe Ansatz (53) with (55) and $K_{1 \ldots n}(\underline{u})=K^{(0)}{ }_{1 \ldots n}(\underline{u})$. 


\subsubsection{The off-shell Bethe Ansatz for $O(3)$}

The R-matrix is given by (5) and (7) for $\nu=2$

$$
R(u)=\mathbf{1}+c(u) P+d(u) K, \quad c(u)=\frac{-1}{u}, d(u)=\frac{1}{u-1 / 2} .
$$

The solution of the difference equations is given by the off-shell Bethe Ansatz 47) with

$$
\psi(u)=\frac{1}{u-1}, \tau(v)=v^{2}
$$

The Bethe vector $\Psi$ is expressed in terms of the co-vectors 43.

$$
\Psi_{\underline{\alpha}}(\underline{u}, \underline{v})=L(\underline{v}) \Phi_{\underline{\alpha}}(\underline{u}, \underline{v}),
$$

where the scalar function $L(\underline{v})$ has to satisfy

$$
\begin{aligned}
& L\left(\ldots, v_{i}, v_{j}, \ldots\right)=L\left(\ldots, v_{j}, v_{i}, \ldots\right) \tilde{\tilde{R}}\left(v_{i j}\right) \\
& L\left(v_{1}^{\prime}, v_{2}, \ldots, v_{m}\right)=L\left(v_{2}, \ldots, v_{m}, v_{1}\right)
\end{aligned}
$$

with $\tilde{R}(v)=\frac{(v+1)(v-1 / 2)}{(v-1)(v+1 / 2)}$ and $v^{\prime}=v+1$. The the minimal solution of these equations is

$$
\begin{aligned}
& L(\underline{v})=\prod_{1 \leq i<j \leq m} L\left(v_{i j}\right) \\
& L(v)=\frac{\pi}{4} \frac{(v-1 / 2)}{v(v-1)} \tan \pi v .
\end{aligned}
$$

The $O(3)$ weight of the state is

$$
w=n-m
$$

\subsubsection{The off-shell Bethe Ansatz for $O(4)$}

The R-matrix is given by (5) and (7) for $\nu=1$

$$
R(u)=\mathbf{1}+c(u) P+d(u) K, \quad c(u)=\frac{-1}{u}, \quad d(u)=\frac{1}{u-1} .
$$

We could apply theorem 3.4 and write the off-shell Bethe Ansatz for $O(4)$ in terms of an $O(2)$ problem. However, the latter cannot be solved by the Bethe Ansatz because the R-matrix is diagonal (note that $R_{1 \overline{1}}^{1 \overline{1}}=0$ ). But there is another way to solve the $O(4)$ problem. The group isomorphism $O(4) \simeq S U(2) \otimes S U(2)$ reflects itselfs in terms of the corresponding R-matrices. Indeed, the $O(4)$ R-matrix can be written as a tensor product of two $S U(2)$ R-matrices, or more precisely

$$
\left(\tilde{R}^{O(4)}\right)_{\alpha \beta}^{\delta \gamma} \Gamma_{A B}^{\alpha} \Gamma_{C D}^{\beta}=\Gamma_{C^{\prime} D^{\prime}}^{\delta} \Gamma_{A^{\prime} B^{\prime}}^{\gamma}\left(\tilde{R}_{+}^{S U(2)}\right)_{A C}^{C^{\prime} A^{\prime}}\left(\tilde{R}_{-}^{S U(2)}\right)_{B D}^{D^{\prime} B^{\prime}}
$$


The $S U(2)$ R-matrices $\tilde{R}_{ \pm}^{S U(2)}(u)=R_{ \pm}^{S U(2)}(u) / a(u)$ correspond to the spinor representations of $O(4)$ with positive (negative) chirality

$$
R_{ \pm}^{S U(2)}=\mathbf{1}+c(u) \mathbf{P}
$$

where the amplitude $c(u)$ is given by (7). The relative R-matrix for states of different chirality is trivial $\tilde{R}=\mathbf{1}$. The intertwiners are

$$
\Gamma_{A B}^{\alpha}=\left(\gamma_{+} \gamma^{\alpha} C\right)_{A B}
$$

with the $O(4)$ gamma matrices $\gamma^{\alpha}, \gamma_{+}=\frac{1}{2}\left(1+\gamma^{5}\right)$ and the charge conjugation matrix $C$. For more details see [28, 29]. In the complex basis of the $O(4)$ and the fundamental representations of the $S U(2)$ the states have the $O(4)$ weights

\begin{tabular}{ccccc} 
vector states & $O(4)$ weights & & spinor states & $O(4)$ weights \\
\cline { 1 - 1 } \cline { 5 - 6 } 1 & $(1,0)$ & & $\uparrow_{+}$ & $\left(\frac{1}{2}, \frac{1}{2}\right)$ \\
2 & $(0,1)$ & & $\downarrow_{+}$ & $\left(-\frac{1}{2},-\frac{1}{2}\right)$ \\
$\overline{2}$ & $(0,-1)$ & & $\uparrow_{-}$ & $\left(\frac{1}{2},-\frac{1}{2}\right)$ \\
$\overline{1}$ & $(-1,0)$ & & $\downarrow_{-}$ & $\left(-\frac{1}{2}, \frac{1}{2}\right)$.
\end{tabular}

Because of weight conservation the intertwiner matrix is diagonal in this basis and is calculated to be

$$
\Gamma_{A B}^{\alpha}=\left(\begin{array}{cccc}
\Gamma_{\uparrow+\uparrow-}^{1} & 0 & 0 & 0 \\
0 & \Gamma_{\uparrow+\downarrow-}^{2} & 0 & 0 \\
0 & 0 & \Gamma_{\downarrow+\uparrow-}^{\overline{2}} & 0 \\
0 & 0 & 0 & \Gamma_{\downarrow+\downarrow-}^{\overline{1}}
\end{array}\right)=\left(\begin{array}{cccc}
-1 & 0 & 0 & 0 \\
0 & 1 & 0 & 0 \\
0 & 0 & 1 & 0 \\
0 & 0 & 0 & 1
\end{array}\right)
$$

We also use the dual intertwiner $\Gamma_{\alpha}^{A B}$ with

$$
\sum_{A, B} \Gamma_{A B}^{\alpha^{\prime}} \Gamma_{\alpha}^{A B}=\delta_{\alpha}^{\alpha^{\prime}}, \sum_{\alpha} \Gamma_{\alpha}^{A^{\prime} B^{\prime}} \Gamma_{A B}^{\alpha}=\delta_{A}^{A^{\prime}} \delta_{B}^{B^{\prime}}
$$

We write the co-vector valued function $K_{\underline{\alpha}}(\underline{u})$ as

$$
K_{\underline{\alpha}}(\underline{u})=K_{\underline{A}}^{(+)}(\underline{u}) K_{\underline{B}}^{(-)}(\underline{u}) \Gamma_{\underline{A}}^{\underline{A}} \underline{B},
$$

where $\Gamma_{\underline{\underline{A}}}^{\underline{A}}=\prod_{i=1}^{n} \Gamma_{\alpha_{i}}^{A_{i} B_{i}}$. The transfer matrix $\operatorname{tr} \tilde{T}^{O(4)}(\underline{u}, v)$ can also be decomposed such that

$$
\begin{aligned}
K_{\underline{\alpha}}(\underline{u})\left(\tilde{T}^{O(4)}\right)_{\gamma}^{\gamma}(\underline{u}, v) & =K_{\underline{\alpha}}(\underline{u}) \Gamma_{A^{\prime} B^{\prime}}^{\gamma}\left(\tilde{T}_{+}^{S U(2)}\right)_{A}^{A^{\prime}}(\underline{u}, v)\left(\tilde{T}_{-}^{S U(2)}\right)_{B}^{B^{\prime}}(\underline{u}, v) \Gamma_{\gamma}^{A B} \\
& =\left(K_{\underline{A}}^{(+)}(\underline{u})\left(\tilde{T}_{+}^{S U(2)}\right)_{A}^{A}(\underline{u}, v)\right)\left(K_{\underline{B}}^{(-)}(\underline{u})\left(\tilde{T}_{-}^{S U(2)}\right)_{B}^{B}(\underline{u}, v)\right) \Gamma_{\underline{\alpha}}^{A} \underline{B},
\end{aligned}
$$

where (59) has been used. Therefore $K_{\underline{\alpha}}(\underline{u})$ satisfies the $O(4)$ symmetry relation (32) and the difference equation 33 if the $K_{\underline{A}}^{( \pm)}(\underline{u})$ satisfy the corresponding $S U(2)$ relations. 
The $S U(2)$ on-shell Bethe Ansatz is well known and the off-shell case has been solved in [14, 6, 30]

$$
\begin{aligned}
K_{\underline{A}}(\underline{u}) & =\sum_{\underline{v}} g(\underline{u}, \underline{v}) \Psi_{\underline{A}}(\underline{u}, \underline{v}) \\
\Psi(\underline{u}, \underline{v}) & =\Omega C\left(\underline{u}, v_{m}\right) \ldots C\left(\underline{u}, v_{1}\right),
\end{aligned}
$$

where $g(\underline{u}, \underline{v})$ is given by $(48)$ and

$$
\psi(u)=\frac{\Gamma\left(-\frac{1}{2}+\frac{u}{2}\right)}{\Gamma\left(\frac{u}{2}\right)}, \tau(v)=v .
$$

The summation over $\underline{v}$ is specified by

$$
\underline{v}=\left(v_{1}, \ldots, v_{m}\right)=\left(\tilde{v}_{1}-2 l_{1}, \ldots, \tilde{v}_{m}-2 l_{m}\right), l_{i} \in \mathbf{Z},
$$

where the $\tilde{v}_{i}$ are arbitrary constants. The $S U(2)$ weights of the state 62 are $w=$ $(n-m, m)$ and due to (57) the $O(4)$ weights are

$$
w= \begin{cases}(n-m,-m) & \text { for positive chirality spinors } \\ (n-m, m) & \text { for negative chirality spinors }\end{cases}
$$

Therefore the $O(4)$ weights of $(60)$ are

$$
w=\left(n-n_{-}-n_{+}, n_{-}-n_{+}\right),
$$

where $n_{ \pm}$are the numbers of positive (negative) chirality $C$-operators.

\section{Weights of off-shell $O(N)$ Bethe vectors}

In this section we analyze some group theoretical properties of off-shell Bethe states. We show that they are highest weight states and we calculate the weights. The first result is not only true for the conventional Bethe Ansatz, which solves an eigenvalue problem and which is well known, but it is also true, as we will show, for the off-shell one which solves a difference equation (or a differential equation).

By the asymptotic expansion of the R-matrix (5) and the monodromy matrix (12) we get for $u \rightarrow \infty$

$$
\begin{aligned}
R_{a b}(u) & =\mathbf{1}_{a b}-\frac{1}{u} M_{a b}+O\left(u^{-2}\right) \\
M_{a b} & =\mathbf{P}_{a b}-\mathbf{K}_{a b} .
\end{aligned}
$$

More explicitly eq. 12 gives

$$
\begin{aligned}
T_{1 \ldots n, a}(\underline{u}, u) & =\mathbf{1}_{1 \ldots n, a}+\frac{1}{u} M_{1 \ldots n, a}+O\left(u^{-2}\right) \\
M_{1 \ldots n, a} & =\left(\mathbf{P}_{1 a}-\mathbf{K}_{1 a}\right)+\cdots+\left(\mathbf{P}_{n a}-\mathbf{K}_{n a}\right) .
\end{aligned}
$$


The matrix elements of $M_{1 \ldots n, a}$, as a matrix in the auxiliary space, are the $O(N)$ Lie algebra generators. In the following we will consider only operators acting in the fixed tensor product space $V=V^{1 \ldots n}$ of (1). Therefore we will omit the indices $1 \ldots n$. In terms of the matrix elements in the auxiliary space $V_{a}$ the generators act on the basis states as

$$
\left\langle\alpha_{1}, \ldots, \alpha_{i}, \ldots, \alpha_{n}\right| M_{\alpha}^{\alpha^{\prime}}=\sum_{i=1}^{n}\left(\delta_{\alpha \alpha_{i}}\left\langle\alpha_{1}, \ldots, \alpha^{\prime}, \ldots, \alpha_{n}\right|-\delta_{\alpha^{\prime} \bar{\alpha}_{i}}\left\langle\alpha_{1}, \ldots, \bar{\alpha}, \ldots, \alpha_{n}\right|\right) .
$$

The diagonal elements of $M_{\alpha}^{\alpha^{\prime}}$ are the the weight operators $W_{\alpha}$ with

$$
\begin{aligned}
\left\langle\alpha_{1}, \ldots, \alpha_{i}, \ldots, \alpha_{n}\right| W_{\alpha} & =w_{\alpha}\left\langle\alpha_{1}, \ldots, \alpha_{i}, \ldots, \alpha_{n}\right| \\
w_{\alpha} & =n_{\alpha}-n_{\bar{\alpha}}
\end{aligned}
$$

where $n_{\alpha}$ is the number of particles $\alpha$ in the state. It is sufficient to consider only the weights

$$
w=\left(w_{1}, \ldots, w_{[N / 2]}\right)
$$

because of $W_{\alpha}=-W_{\bar{\alpha}}$ and $\langle\underline{\alpha}| W_{0}=0$ for $N$ odd.

The Yang-Baxter relations (14) yield for $u_{a} \rightarrow \infty$

$$
\left[M_{a}+M_{a b}, T_{b}\left(u_{b}\right)\right]=0
$$

and if additionally $u_{b} \rightarrow \infty$, we get

$$
\left[M_{a}+M_{a b}, M_{b}\right]=0
$$

or for the matrix elements (in the real basis)

$$
\begin{aligned}
{\left[M_{\alpha}^{\alpha^{\prime}}, T_{\beta}^{\beta^{\prime}}(u)\right] } & =-\delta_{\alpha}^{\beta^{\prime}} T_{\beta}^{\alpha^{\prime}}(u)+\delta^{\alpha^{\prime} \beta^{\prime}} T_{\beta}^{\alpha}(u)+T_{\alpha}^{\beta^{\prime}}(u) \delta_{\beta}^{\alpha^{\prime}}-T_{\alpha^{\prime}}^{\beta^{\prime}}(u) \delta_{\alpha \beta} \\
{\left[M_{\alpha}^{\alpha^{\prime}}, M_{\beta}^{\beta^{\prime}}\right] } & =-\delta_{\alpha}^{\beta^{\prime}} M_{\beta}^{\alpha^{\prime}}+\delta^{\alpha^{\prime} \beta^{\prime}} M_{\beta}^{\alpha}+M_{\alpha}^{\beta^{\prime}} \delta_{\beta}^{\alpha^{\prime}}-M_{\alpha^{\prime}}^{\beta^{\prime}} \delta_{\alpha \beta} .
\end{aligned}
$$

Equation (74) represents the structure relations of the $O(N)$ Lie algebra and (73) the $O(N)$-covariance of $T$. In particular the transfer matrix is invariant

$$
\left[M_{\alpha}^{\alpha^{\prime}}, \operatorname{tr} T(u)\right]=0
$$

Theorem 4.1 1. If the co-vector valued function

$$
K_{\underline{\alpha}}(\underline{u})=\sum_{\underline{v}} g(\underline{u}, \underline{v}) L_{\underline{\beta}}(\underline{v}) \Phi_{\underline{\alpha}}^{\stackrel{\leftrightarrow}{\beta}}(\underline{u}, \underline{v})
$$

is given by the nested off-shell Bethe Ansatz (53) the weights (70) are $w=$

$$
\left(w_{1}, \ldots, w_{[N / 2]}\right)= \begin{cases}\left(n-n_{1}, \ldots, n_{[N / 2]-1}-n_{[N / 2]}\right) & \text { for } N \text { odd } \\ \left(n-n_{1}, \ldots, n_{[N / 2]-2}-n_{-}-n_{+}, n_{-}-n_{+}\right) & \text {for } N \text { even } .\end{cases}
$$


2. If $K_{\underline{\alpha}}(\underline{u})$ satisfies the conditions of theorem 3.4 and if $L_{\underline{\beta}}(\underline{v})$ is a highest weight state, then $K_{\underline{\alpha}}(\underline{u})$ is a highest weight state:

$$
K(\underline{u}) M_{\alpha}^{\alpha^{\prime}}=0 \text { for } \alpha^{\prime}<\alpha .
$$

(Recall that $\alpha^{\prime}<\alpha$ is to be understood corresponding to the ordering $1,2, \ldots,[N / 2],(0), \overline{[N / 2]}, \ldots, \overline{2}, \overline{1}$.)

3. The weights satisfy the highest weight condition

$$
\left\{\begin{array}{l}
w_{1} \geq w_{2} \geq \cdots \geq w_{[N / 2]} \geq 0 \quad \text { for } \quad N \text { odd } \\
w_{1} \geq w_{2} \geq \cdots \geq\left|w_{[N / 2]}\right| \quad \text { for } \quad N \text { even } .
\end{array}\right.
$$

The proof of this theorem can be found in appendix C. We mention that for $N$ even the highest weight property was already discussed in appendix B of [17].

\section{$5 \quad$ Examples}

In this section we present some explicit examples of the solutions of the $O(N)$ - difference equations.

Example 5.1 The simplest example is obtained for $k_{\max }=1$ which means the trivial solution of the difference equations

$$
K_{\underline{\alpha}}=\Omega_{\underline{\alpha}}=\delta_{\alpha_{1}}^{1} \ldots \delta_{\alpha_{n}}^{1} .
$$

The weights of $K_{\underline{\alpha}}$ are $w=(n, 0, \ldots, 0)$. In the language of spin chains this case corresponds to the ferromagnetic ground state.

Example 5.2 For $N \neq 4, k_{\max }=2$ and $n^{(1)}=1$ the solution reads

$$
K_{\underline{\alpha}}(\underline{u})=\sum_{v} g(\underline{u}, v) L_{\dot{\beta}}(v)\left(\Omega C^{\stackrel{\leftrightarrow}{\beta}}(\underline{u}, v)\right)_{\underline{\alpha}}
$$

with $v=\tilde{v}-2 l / \nu(l \in \mathbf{Z}, \tilde{v}$ an arbitrary constant $)$ and

$$
\begin{gathered}
g(\underline{u}, v)=\prod_{i=1}^{n} \psi\left(u_{i}-v\right), \psi(u)=\frac{\Gamma\left(-\frac{1}{2} \nu+\frac{u}{2} \nu\right)}{\Gamma\left(\frac{u}{2} \nu\right)} \\
L_{\dot{\beta}}(v)=\delta_{\dot{\beta}}^{2} \text { for } N>4 \text { and }=\delta_{\dot{\beta}}^{0} \text { for } N=3 .
\end{gathered}
$$

The weights of this co-vector $K_{\underline{\alpha}}(\underline{u})$ are $w=(n-1,1,0, \ldots, 0)$. The action of the operator $C^{\dot{\beta}}(\underline{u}, v)$ on the reference state is easily calculated with the help of eqs. (6) (12) and (13).

As a particular case of this example we determine explicitly the solution for the following 
Example 5.3 The action of the $C$-operator on the reference state for the case of $n=2$ and $N>4$ of Example 5.2 yields

$$
\left(\Omega C^{2}(\underline{u}, v)\right)_{\underline{\alpha}}=\delta_{\alpha_{1}}^{2} \delta_{\alpha_{2}}^{1} c\left(u_{1}-v\right) a\left(u_{2}-v\right)+\delta_{\alpha_{1}}^{1} \delta_{\alpha_{2}}^{2} c\left(u_{2}-v\right) .
$$

Therefore we obtain (with the help of Dougall's formula)

$$
\begin{aligned}
& K_{\underline{\alpha}}(\underline{u})=\sum_{v} \psi\left(u_{1}-v\right) \psi\left(u_{2}-v\right)\left(\delta_{\alpha_{1}}^{2} \delta_{\alpha_{2}}^{1} c\left(u_{1}-v\right) a\left(u_{2}-v\right)+\delta_{\alpha_{1}}^{1} \delta_{\alpha_{2}}^{2} c\left(u_{2}-v\right)\right) \\
& \quad=\frac{\pi^{2} \Gamma(\nu) /\left(\Gamma\left(\frac{1}{2} \nu\right)\right)^{2}}{\sin \frac{1}{2} \pi \nu\left(1-u_{1}+\tilde{v}\right) \sin \frac{1}{2} \pi \nu\left(1-u_{2}+\tilde{v}\right)} \frac{\delta_{\alpha_{1}}^{\stackrel{\beta}{\beta}} \delta_{\alpha_{2}}^{1}-\delta_{\alpha_{1}}^{1} \delta_{\alpha_{2}}^{\dot{\beta}}}{\Gamma\left(1+\frac{1}{2} \nu\left(u_{21}+1\right)\right) \Gamma\left(-\frac{1}{2} \nu\left(u_{21}-1\right)\right)} .
\end{aligned}
$$

The weights are $w=(1,1,0, \ldots, 0)$.

Example 5.4 The special case $N=3$ gives

$$
K_{\underline{\alpha}}(\underline{u})=\frac{-\pi}{\sin \pi\left(-u_{1}+\tilde{v}\right) \sin \pi\left(-u_{2}+\tilde{v}\right)} \frac{\sin \pi u_{12}}{u_{12}\left(u_{12}-1\right)}\left(\delta_{\alpha_{1}}^{0} \delta_{\alpha_{2}}^{1}-\delta_{\alpha_{1}}^{1} \delta_{\alpha_{2}}^{0}\right)
$$

and weight $w=(1)$.

Example 5.5 To obtain the results in examples 5.2 and 5.3 for $N=4$ we have to use the corresponding $S U(2)$ formulas with $\psi(u)=\Gamma\left(-\frac{1}{2}+\frac{u}{2}\right) / \Gamma\left(\frac{u}{2}\right)$

$$
K_{\underline{A}}^{( \pm)}(\underline{u})=\sum_{v} g(\underline{u}, v)(\Omega C(\underline{u}, v))_{\underline{A}} .
$$

For $n=2$

$$
\begin{aligned}
K_{\underline{A}}^{( \pm)}(\underline{u}) & =\sum_{v} \psi\left(u_{1}-v\right) \psi\left(u_{2}-v\right)\left(\delta_{A_{1}}^{\downarrow} \delta_{A_{2}}^{\uparrow} c\left(u_{1}-v\right) a\left(u_{2}-v\right)+\delta_{A_{1}}^{\uparrow} \delta_{A_{2}}^{\downarrow} c\left(u_{2}-v\right)\right) \\
& =K^{( \pm)}(\underline{u})\left(\delta_{A_{1}}^{\downarrow} \delta_{A_{2}}^{\uparrow}-\delta_{A_{1}}^{\uparrow} \delta_{A_{2}}^{\downarrow}\right) \\
K^{( \pm)}(\underline{u}) & =\pi \frac{1}{\sin \frac{1}{2} \pi\left(1-u_{1}+\tilde{v}^{( \pm)}\right) \sin \frac{1}{2} \pi\left(1-u_{2}+\tilde{v}^{( \pm)}\right)} \\
& \times \frac{1}{\Gamma\left(1+\frac{1}{2}\left(u_{21}+1\right)\right) \Gamma\left(-\frac{1}{2}\left(u_{21}-1\right)\right)} .
\end{aligned}
$$

There are three possibilities: using the intertwiners (58) we find

$$
\begin{aligned}
K_{\underline{\alpha}}(\underline{u}) & =K_{\underline{A}}^{(+)}(\underline{u}) \Omega_{\underline{B}}^{(-)} \Gamma \underline{A B}=K^{(+)}(\underline{u})\left(\delta_{A_{1}}^{\downarrow} \delta_{A_{2}}^{\uparrow}-\delta_{A_{1}}^{\uparrow} \delta_{A_{2}}^{\downarrow}\right)\left(\delta_{B_{1}}^{\uparrow} \delta_{B_{2}}^{\uparrow}\right) \Gamma_{\underline{\alpha}}^{\underline{A}} \underline{B} \\
& =K^{(+)}(\underline{u})\left(-\delta_{\alpha_{1}}^{\overline{2}} \delta_{\alpha_{2}}^{1}+\delta_{\alpha_{1}}^{1} \delta_{\alpha_{1}}^{\overline{2}}\right)
\end{aligned}
$$


with weights $w=(1,-1)$, and

$$
\begin{aligned}
K_{\underline{\alpha}}(\underline{u}) & =\Omega_{\underline{A}}^{(+)}(\underline{u}) K_{\underline{B}}^{(-)}(\underline{u}) \Gamma \underline{A} \underline{\underline{\alpha}}=\left(\delta_{A_{1}}^{\uparrow} \delta_{A_{2}}^{\uparrow}\right)\left(\delta_{B_{1}}^{\downarrow} \delta_{B_{2}}^{\uparrow}-\delta_{B_{1}}^{\uparrow} \delta_{B_{2}}^{\downarrow}\right) K^{(-)}(\underline{u}) \Gamma_{\underline{\alpha}}^{\underline{A}} \underline{\underline{B}} \\
& =K^{(-)}(\underline{u})\left(-\delta_{\alpha_{1}}^{2} \delta_{\alpha_{2}}^{1}+\delta_{\alpha_{1}}^{1} \delta_{\alpha_{2}}^{2}\right)
\end{aligned}
$$

with weights $w=(1,1)$, and

$$
\begin{aligned}
K_{\underline{\alpha}}(\underline{u}) & =K_{\underline{A}}^{(+)}(\underline{u}) K_{\underline{B}}^{(-)} \Gamma_{\underline{\alpha}}^{A B} \\
& =K^{(+)}(\underline{u}) K^{(-)}(\underline{u})\left(\delta_{A_{1}}^{\downarrow} \delta_{A_{2}}^{\uparrow}-\delta_{A_{1}}^{\uparrow} \delta_{A_{2}}^{\downarrow}\right)\left(\delta_{B_{1}}^{\downarrow} \delta_{B_{2}}^{\uparrow}-\delta_{B_{1}}^{\uparrow} \delta_{B_{2}}^{\downarrow}\right) \Gamma_{\underline{\alpha}}^{\underline{A}} \underline{B} \\
& =K^{(+)}(\underline{u}) K^{(-)}(\underline{u})\left(-\delta_{\alpha_{1}}^{\overline{1}} \delta_{\alpha_{2}}^{1}-\delta_{\alpha_{1}}^{2} \delta_{\alpha_{2}}^{\overline{2}}-\delta_{\alpha_{1}}^{\overline{2}} \delta_{\alpha_{2}}^{2}-\delta_{\alpha_{1}}^{1} \delta_{\alpha_{2}}^{\overline{1}}\right)
\end{aligned}
$$

with weights $w=(0,0)$.

\section{Conclusion}

In this article we solved the $O(N)$-matrix difference equations by means of the off-shell algebraic nested Bethe Ansatz. We introduced a new object called $\Pi$-matrix to overcome the difficulties connected to the special peculiarities of the $O(N)$ symmetric R-matrix structure. The highest weights properties of the solutions were analyzed. We believe that our construction can also be applied to the cases with similar group theoretical complexities, such as $B_{n}, C_{n}, D_{n}$ Lie algebras and superalgebra $O \operatorname{sp}(n \mid 2 m)$ (see [16]).

\section{Acknowledgments}

The authors have profited from discussions with A. Fring, R. Schrader, F. Smirnov and A. Belavin. H.B. thanks R. Flume, R. Poghossian and P. Wiegmann for valuable discussions. H.B. and M.K. were supported by Humboldt Foundation and H.B. is also supported by the Armenian grant 11-1_c028. A.F. acknowledges support from DAAD (Deutscher Akademischer Austausch Dienst) and CNPq (Conselho Nacional de Desenvolvimento Científico e Tecnológico).

\section{Appendix}

\section{A Proof of Lemma 2.5}

\section{Proof.}

(a) We prove (23) by induction: It is true for $m=2$, because similar to (21)

$$
\pi_{1} \pi_{2} \bar{e}_{b} T_{12, b}\left(\underline{u}, u_{b}\right) \bar{e}^{b}=\pi_{1} \pi_{2}+f\left(u_{12}\right) \stackrel{\circ}{\mathbf{C}}^{12} \bar{e}_{1} e_{2} .
$$


We assume (23) for $m-1$ and replace in the definition (20) $\Pi_{2 \ldots m}$ as given by (23)

$$
\begin{aligned}
\Pi_{1 \ldots m} & =\pi_{1} \Pi_{2 \ldots m} \bar{e}_{a} T_{1 \ldots m, a} \bar{e}^{a} \\
& =\pi_{1}\left(\Pi_{2 \ldots m-1} \pi_{m} \bar{e}_{b} T_{2 \ldots m, b} \bar{e}^{b}\right) \bar{e}_{a} T_{1 \ldots m, a} \bar{e}^{a} \\
& =\left(\pi_{1} \Pi_{2 \ldots m-1} \pi_{m}\right) \bar{e}_{b} T_{1 \ldots m b b} \bar{e}^{b} \bar{e}_{a} T_{1 \ldots m, a} \bar{e}^{a} \\
& =\left(\pi_{1} \Pi_{2 \ldots m-1} \pi_{m}\right) \bar{e}_{a} T_{1 \ldots m, a} \bar{e}^{a} \bar{e}_{b} T_{1 \ldots m, b} \bar{e}^{b} \\
& =\left(\pi_{1} \Pi_{2 \ldots m-1} \pi_{m}\right) \bar{e}_{a} T_{1 \ldots m-1, a} \bar{e}^{a} \bar{e}_{b} T_{1 \ldots m, b} \bar{e}^{b} \\
& =\left(\Pi_{1 \ldots m-1} \pi_{m}\right) \bar{e}_{b} T_{1 \ldots m b b} \bar{e}^{b} .
\end{aligned}
$$

In equality 3 we have $T_{2 \ldots m, b}$ replaced by $T_{1 \ldots m, b}$, because

$$
\bar{e}_{b} R_{1 b}\left(u_{1 b}\right) \bar{e}_{a} R_{1 a}\left(u_{1 a}\right)=\bar{e}_{b} \bar{e}_{a} R_{1 a}(1)+c\left(u_{1 b}\right) \bar{e}_{1} \bar{e}_{a} R_{1 a}(1)=\bar{e}_{b} \bar{e}_{a} R_{1 a}(1)
$$

holds, where $\bar{e}_{1} \bar{e}_{a} R_{1 a}(1)=\bar{e}_{1} \bar{e}_{a} a(1)=0$ has been used. Similarly, equality 5 holds. Equality 4 holds because the Yang Baxter equation for $R$ implies that $\bar{e}_{a} T_{1 \ldots m, a} \bar{e}^{a}$ and $\bar{e}_{b} T_{1 \ldots m, b} \bar{e}^{b}$ commute.

(b) Again we prove (24) by induction. For $m=2$ the claim was proved in section 2.4. for $m>2$ it follows for $1<i<j$ from (20) and for $i<j<m$ from (23).

(c) The proof of equation (25) is similar to that of a). We commute $T\left(u_{a}\right)$ and $T\left(u_{0}\right)$, use $\bar{e}_{0} \pi_{1} R_{10}\left(u_{10}\right) R_{1 a}(1)=\bar{e}_{0} \pi_{1}$ and apply induction:

$$
\begin{aligned}
\Pi_{1 \ldots m} \bar{e}_{0} T_{1 \ldots m, 0}\left(u_{0}\right) \bar{e}^{0} & =\left(\pi_{1} \Pi_{2 \ldots m}\right) \bar{e}_{a} T_{1 \ldots m, a} \bar{e}^{a} \bar{e}_{0} T_{1 \ldots m, 0}\left(u_{0}\right) \bar{e}^{0} \\
& =\left(\pi_{1} \Pi_{2 \ldots m}\right) \bar{e}_{0} T_{1 \ldots m, 0}\left(u_{0}\right) \bar{e}^{0} \bar{e}_{a} T_{1 \ldots m, a} \bar{e}^{a} \\
& =\pi_{1}\left(\Pi_{2 \ldots m}\right) \bar{e}_{0} T_{2 \ldots m, 0}\left(u_{0}\right) \bar{e}^{0} \bar{e}_{a} T_{1 \ldots m, a} \bar{e}^{a} \\
& =\left(\pi_{1} \Pi_{2 \ldots m}\right) \bar{e}_{a} T_{1 \ldots m, a} \bar{e}^{a}=\Pi_{1 \ldots m} .
\end{aligned}
$$

(d) Equations (26) and 27) follow from 20 and $R_{1 \alpha}^{\overline{1} \stackrel{\alpha}{\alpha}_{1}}(1)=0, R_{\alpha \alpha}^{\overline{1} \stackrel{\alpha}{\alpha}_{1}}(1)=\delta_{\alpha}^{\alpha_{\alpha}} \delta_{\alpha}^{\overline{1}}$ and analogously (28) and (29).

\section{B Proof of the main theorem 3.4}

In the following we use the convention that $\alpha$ etc. take the values $1,2, \ldots,(0), \ldots, \overline{2}, \overline{1}$ and $\stackrel{\circ}{\alpha}$ etc. take the values $2, \ldots,(0), \ldots, \overline{2}$.

$$
K_{\underline{\alpha}}(\underline{u})=\sum_{\underline{v}} g(\underline{u}, \underline{v}) L_{\underline{\beta}}(\underline{v}) \Pi_{\underline{\beta}}^{\stackrel{\circ}{\beta}}(\underline{v})\left(\Omega T_{1}^{\beta_{m}}\left(\underline{u}, v_{m}\right) \ldots T_{1}^{\beta_{1}}\left(\underline{u}, v_{1}\right)\right)_{\underline{\alpha}} .
$$


(i) Proof. Property (i) in the form of (32) follows directly from the Yang-Baxter equations and the action of the R-matrix on the pseudo-ground state $\Omega$

$$
\begin{aligned}
\left(T_{\ldots j i \ldots}\right)_{1}^{\beta}\left(\ldots u_{j}, u_{i} \ldots\right) R_{i j}\left(u_{i j}\right) & =R_{i j}\left(u_{i j}\right)\left(T_{\ldots i j \ldots}\right)_{1}^{\beta}\left(\ldots u_{i}, u_{j} \ldots\right) \\
\Omega_{\ldots i j \ldots} R_{i j}\left(u_{i j}\right) & =a\left(u_{i j}\right) \Omega_{\ldots i j \ldots} .
\end{aligned}
$$

(ii) Proof. We prove

$$
K_{1 \ldots n}\left(\underline{u}^{\prime}\right)=K_{1 \ldots n}(\underline{u}) Q_{1 \ldots n}(\underline{u}),
$$

where $\underline{u}^{\prime}=\left(u_{1}+2 / \nu, u_{2} \ldots, u_{n}\right)$. The matrix $Q(\underline{u})=Q(\underline{u}, 1)$ is given by (38). Note that we assign to the auxiliary space of $\tilde{T}_{Q}(\underline{u})$ the spectral parameter $u_{1}$ on the right hand side and $u_{1}^{\prime}=u_{1}+2 / \nu$ on the left hand side. In the following we will suppress the indices $1 \ldots n$. We are now going to prove $(B .1)$ in the form

$$
K(\underline{u})\left(A_{Q}(\underline{u})+D_{Q_{\dot{\beta}}^{\circ}}^{\stackrel{\leftrightarrow}{u}}(\underline{u})+A_{3, Q}(\underline{u})\right)=\prod_{k=2}^{n} a\left(u_{k 1}\right) K\left(\underline{u}^{\prime}\right),
$$

where $K(\underline{u})$ is a co-vector valued function as given by eq. (47) and the Bethe Ansatz state (40). To analyze the left hand side of eq. $(\overline{B .2})$ we proceed as usual in the algebraic Bethe Ansatz and push $A_{Q}(\underline{u}), D_{Q_{\dot{\beta}}^{\circ}}^{\stackrel{\leftrightarrow}{u}}(\underline{u})$ and $A_{3, Q}(\underline{u})$ through all the $T_{1}^{\beta_{i}}$-operators. As usual we obtain wanted terms and unwanted terms. We first find that the wanted contribution from $A_{Q}(\underline{u})$ already gives the result we are looking for. Secondly the wanted contributions from $D_{Q_{\dot{\beta}}^{\prime}}^{\stackrel{\dot{\beta}}{\beta}}(\underline{u})$ and $A_{3, Q}(\underline{u})$ applied to $\Omega$ give zero because of 46 . Thirdly the unwanted contributions from $A_{Q}(\underline{u}), D_{Q_{\dot{\beta}}^{\circ}}^{\stackrel{\leftrightarrow}{u}}(\underline{u})$ and $A_{3, Q}(\underline{u})$ can be written as differences which cancel after summation over the $v_{j}$. All these three facts can be seen as follows.

The "wanted terms" from $A_{Q}$ are obtained if one writes the Zapletal commutations rule (35) as

$$
T_{1}^{\beta_{k}}\left(\underline{u}, v_{k}\right) A_{Q}(\underline{u})=A_{Q}(\underline{u}) T_{1}^{\beta_{i}}\left(\underline{u}^{\prime}, v_{k}\right) a\left(u_{1}-v_{k}\right)+u w .
$$

Therefore we obtain

$$
(L(\underline{v}) \Pi(\underline{u}, \underline{v}))_{\underline{\beta}} \Omega T_{1}^{\beta_{m}}\left(\underline{u}, v_{m}\right) \cdots T_{1}^{\beta_{1}}\left(\underline{u}, v_{1}\right) A_{Q}(\underline{u})=w^{A}+u w^{A}
$$

with

$$
\begin{aligned}
& w^{A}(\underline{u}, \underline{v})=(L(\underline{v}) \Pi(\underline{u}, \underline{v}))_{\underline{\beta}} \Omega A_{Q}(\underline{u}) T_{1}^{\beta_{m}}\left(\underline{u}^{\prime}, v_{m}\right) \cdots T_{1}^{\beta_{1}}\left(\underline{u}^{\prime}, v_{1}\right) \prod_{k=1}^{m} a\left(u_{1}-v_{k}\right) \\
& =\prod_{k=2}^{n} a\left(u_{k 1}\right) \prod_{k=1}^{m} a\left(u_{1}-v_{k}\right) L_{\dot{\beta}}(\underline{v}) \Phi^{\underline{\hat{\beta}}}\left(\underline{u}^{\prime}, \underline{v}\right)
\end{aligned}
$$


and similarly $w^{D}=w^{A_{3}}=0$, because of 46 . Inserted into 47 this yields

$$
\begin{aligned}
(K(\underline{u}) Q)^{\text {wanted }}(\underline{u}) & =\sum_{\underline{v}} g(\underline{u}, \underline{v}) \prod_{k=1}^{m} a\left(u_{1}-v_{k}\right) L_{\dot{\beta}}(\underline{v}) \Phi^{\underline{\dot{\beta}}}\left(\underline{u}^{\prime}, \underline{v}\right) \\
& =\sum_{\underline{v}} g\left(\underline{u}^{\prime}, \underline{v}\right) L_{\dot{\beta}}(\underline{v}) \Phi^{\underline{\hat{\beta}}}\left(\underline{u}^{\prime}, \underline{v}\right)=K\left(\underline{u}^{\prime}\right)
\end{aligned}
$$

because

$$
g(\underline{u}, \underline{v}) \prod_{k=1}^{m} a\left(u_{1}-v_{k}\right)=g\left(\underline{u}^{\prime}, \underline{v}\right),
$$

where (49) has been used. Therefore it remains to prove that the unwanted terms cancel. This will follow from the lemma below.

We apply $Q_{1 \ldots n}(\underline{u})$ to the state $\Psi_{1 \ldots n}(\underline{u}, \underline{v})$ (suppressing the quantum space indices)

$$
\Psi(\underline{u}, \underline{v})\left(A_{Q}(\underline{u})+D_{Q_{\dot{\beta}}^{\circ}}^{\stackrel{\circ}{\beta}}(\underline{u})+A_{3, Q}(\underline{u})\right)=\text { wanted }+ \text { unwanted } .
$$

The wanted contribution has been calculated above and the unwanted terms may be written as (see subsection B.1)

$$
\begin{aligned}
& \text { unwanted }=u w^{A}+u w^{D}+u w^{A_{3}} \\
& u w^{A}=\sum_{i=1}^{m}\left(u w_{C}^{A, i}\right)_{\dot{\gamma}} C_{Q}^{\grave{\gamma}}+\sum_{i=1}^{m} \sum_{\substack{j=1 \\
j \neq i}}^{m} u w_{C_{2}}^{A, i j} C_{2, Q} \\
& u w^{D}=\sum_{i=1}^{m}\left(u w_{C}^{D, i}\right)_{\dot{\gamma}} C_{Q}^{\grave{\gamma}}+\sum_{i=1}^{m} \sum_{\substack{j=1 \\
j \neq i}}^{m} u w_{C_{2}}^{D, i j} C_{2, Q}+\sum_{i=1}^{m}\left(u w_{C_{3}}^{D, i}\right)_{\dot{\gamma}}\left(C_{3, Q}\right)_{\dot{\gamma}^{\prime}} \stackrel{\circ}{\mathbf{\gamma}}^{\dot{\gamma}^{\prime} \hat{\gamma}} \\
& u w^{A_{3}}=\sum_{i=1}^{m} \sum_{\substack{j=1 \\
j \neq i}}^{m} u w_{C_{2}}^{A_{3}, i j} C_{2, Q}+\sum_{i=1}^{m}\left(u w_{C_{3}}^{A_{3}, i}\right)_{\dot{\gamma}}\left(C_{3, Q}\right)_{\dot{\gamma}^{\prime}}{\stackrel{\circ}{\mathbf{C}^{\prime}}}^{\gamma^{\prime} \gamma}
\end{aligned}
$$

Lemma B.1 The unwanted terms satisfy the relations

$$
\begin{aligned}
& \left(u w_{C}^{D, i}\right)_{\dot{\gamma}}(\underline{u}, \underline{v}) g(\underline{u}, \underline{v})=-\left(u w_{C}^{A, i}\right)_{\dot{\gamma}}\left(\underline{u}, \underline{v}^{(i)}\right) g\left(\underline{u}, \underline{v}^{(i)}\right) \\
& \left(u w_{C_{3}}^{A_{3}, i}\right)_{\dot{\gamma}}(\underline{u}, \underline{v}) g(\underline{u}, \underline{v})=-\left(u w_{C_{3}}^{D, i}\right)_{\dot{\gamma}}\left(\underline{u}, \underline{v}^{(i)}\right) g\left(\underline{u}, \underline{v}^{(i)}\right) \\
& u w_{C_{2}}^{D, i j}\left(\underline{u}_{\underline{v}}^{(j)}\right) g\left(\underline{u}, \underline{v}^{(j)}\right)=-u w_{C_{2}}^{A, i j}\left(\underline{u}, \underline{v}^{(i j)}\right) g\left(\underline{u}, \underline{v}^{(i j)}\right)-u w_{C_{2}}^{A_{3}, i j}(\underline{u}, \underline{v}) g(\underline{u}, \underline{v})
\end{aligned}
$$

with the notation

$$
\begin{aligned}
\underline{v} & =v_{1}, \ldots, v_{m} \\
\underline{v}^{(i)} & =v_{1}, \ldots, v_{i}^{\prime}, \ldots, v_{m} \\
\underline{v}^{(i j)} & =v_{1}, \ldots, v_{i}^{\prime}, \ldots, v_{j}^{\prime}, \ldots, v_{m} \\
v^{\prime} & =v+2 / \nu .
\end{aligned}
$$


Equations (B.4) - (B.6) imply that all unwanted terms cancel after summation over the $\underline{v}$ in the Jackson-type Integral (47).

Proof. We can calculate the following unwanted contributions explicitly (see subsection B.1)

$$
\begin{aligned}
& \left(u w_{C}^{A, i}\right)_{\dot{\gamma}}(\underline{u}, \underline{v})=-c\left(u_{1}^{\prime}-v_{i}\right) X_{\dot{\gamma}}^{(i)}(\underline{u}, \underline{v}) \\
& \left(u w_{C}^{D, i}\right)_{\dot{\gamma}}(\underline{u}, \underline{v})=-c\left(v_{i}-u_{1}\right) X_{\dot{\gamma}}^{(i)}\left(\underline{u}, \underline{v}^{(i)}\right) \chi_{i}(\underline{u}, \underline{v}) \\
& \left(u w_{C_{3}}^{D, i}\right)_{\dot{\gamma}}(\underline{u}, \underline{v})=-f\left(u_{1}^{\prime}-v_{i}\right) X_{\dot{\gamma}}^{(i)}(\underline{u}, \underline{v}) \\
& \left(u w_{C_{3}}^{A_{3}, i}\right)_{\dot{\gamma}}(\underline{u}, \underline{v})=f\left(u_{1}-v_{i}\right) X_{\dot{\gamma}}^{(i)}\left(\underline{u}, \underline{v}^{(i)}\right) \chi_{i}(\underline{u}, \underline{v})
\end{aligned}
$$

with

$$
X_{\dot{\gamma}}^{(i)}(\underline{u}, \underline{v})=L\left(v_{i}, \underline{v}_{i}\right)_{\dot{\gamma} \underline{\beta}_{i}} \Phi^{\stackrel{\beta}{\beta}_{i}}\left(\underline{u}, \underline{v}_{i}\right) \prod_{\substack{k=1 \\ k \neq i}}^{m} a\left(v_{i k}\right) a_{1}\left(\underline{u}, v_{i}\right)
$$

and

$$
\chi_{i}(\underline{u}, \underline{v})=\frac{a_{2}\left(\underline{u}, v_{i}\right)}{a_{1}\left(\underline{u}, v_{i}^{\prime}\right)} \prod_{\substack{k=1 \\ k \neq i}}^{m} \frac{a\left(v_{k i}\right)}{a\left(v_{i k}^{\prime}\right)},
$$

where $a_{1}$ and $a_{2}$ are defined in (45). The remaining unwanted terms are

$$
\begin{aligned}
u w_{C_{2}}^{A, i j}(\underline{u}, \underline{v}) & =-c\left(u_{1}^{\prime}-v_{i}\right) X^{(i j)}(\underline{v}) \\
u w_{C_{2}}^{D, i j}(\underline{u}, \underline{v}) & =\left(c\left(u_{1}-v_{i}\right)+f\left(u_{1}^{\prime}-v_{j}\right)\right) X^{(i j)}\left(\underline{v}^{(i)}\right) \chi_{i}(\underline{u}, \underline{v}) \\
u w_{C_{2}}^{A_{3}, i j}(\underline{u}, \underline{v}) & =-f\left(u_{1}-v_{j}\right) X^{(i j)}\left(v_{i}^{\prime}, v_{j}^{\prime}, \underline{v}_{i j}\right) \chi_{i}\left(\underline{u}, \underline{v}^{(j)}\right) \chi_{j}(\underline{u}, \underline{v})
\end{aligned}
$$

with

$$
X^{(i j)}(\underline{u}, \underline{v})=f\left(v_{i j}\right) a\left(v_{i j}\right) L\left(v_{i}, v_{j}, \underline{v}_{i j}\right) \stackrel{\mathrm{C}}{ }^{i j} \Phi\left(\underline{u}, \underline{v}_{i j}\right) \prod_{k=1, k \neq i, j}^{m}\left(a\left(v_{i k}\right) a\left(v_{j k}\right)\right) a_{1}\left(v_{i}\right) a_{1}\left(v_{j}\right) .
$$

The claims B.4 - B.6 follow from the shift property of the function $g(\underline{u}, \underline{v})$ defined in (48)

$$
\chi_{i}(\underline{u}, \underline{v}) g(\underline{u}, \underline{v})=g\left(\underline{u}, \underline{v}^{(i)}\right)
$$

which is due to the shift properties 49 of the functions $\psi(v)$ and $\tau(v)$.

Note that for the on-shell Bethe Ansatz the equations for the unwanted terms are quite similar (apart from the fact that there are no shifts) and their cancellation is equivalent to the Bethe Ansatz equations

$$
\chi_{i}(\underline{u}, \underline{v})=\frac{a_{2}\left(\underline{u}, v_{i}\right)}{a_{1}\left(\underline{u}, v_{i}\right)} \prod_{\substack{k=1 \\ k \neq i}}^{m} \frac{a\left(v_{k i}\right)}{a\left(v_{i k}\right)}=1 .
$$




\section{B.1 The unwanted terms}

The unwanted terms are derived using lemma 2.2. Here we only sketch the derivation of $u w^{A}$ as an example (more details will be published elsewhere [31]). We start with (B.3) use Yang-Baxter relations

$$
\begin{aligned}
w^{A} & =(L \Pi)_{\underline{\beta}} \Omega A_{Q} T_{1}^{\beta_{m}}\left(\underline{u}^{\prime}, v_{m}\right) \cdots T_{1}^{\beta_{1}}\left(\underline{u}^{\prime}, v_{1}\right) a\left(u_{1}-v_{m}\right) \ldots a\left(u_{1}-v_{1}\right) \\
& =(L \Pi)_{\underline{\beta}} \Omega\left(R_{0 m}\left(u_{1}^{\prime}-v_{m}\right) \ldots R_{01}\left(u_{1}^{\prime}-v_{1}\right)\right)_{\gamma, \underline{\beta}}^{\underline{\beta}, 1} T_{1}^{\beta_{m}^{\prime}}\left(\underline{u}, v_{m}\right) \cdots T_{1}^{\beta_{1}^{\prime}}\left(\underline{u}, v_{1}\right)\left(T_{Q}\right)_{1}^{\gamma}
\end{aligned}
$$

and lemma 2.2 in the form of 16

$$
\begin{aligned}
w^{A} & =(L \Pi)_{\underline{\beta}} \Omega T_{1}^{\beta_{m}}\left(z_{m}\right) \cdots T_{1}^{\beta_{1}}\left(z_{1}\right) A_{Q}+\sum_{i=1}^{m} c\left(u_{1}^{\prime}-v_{i}\right) \\
& \times(L \Pi)_{\underline{\beta}} \Omega\left(R_{0 m}\left(v_{i m}\right) \cdots \mathbf{P}_{0 i} \ldots R_{01}\left(v_{i 1}\right)\right)_{\gamma, \underline{\beta}^{\prime}}^{\underline{\beta}, 1} T_{1}^{\beta_{m}^{\prime}}\left(\underline{u}, v_{m}\right) \cdots T_{1}^{\beta_{1}^{\prime}}\left(\underline{u}, v_{1}\right)\left(T_{Q}\right)_{1}^{\gamma} \\
& =(L \Pi)_{\underline{\beta}} \Omega T_{1}^{\beta_{m}}\left(v_{m}\right) \cdots T_{1}^{\beta_{1}}\left(v_{1}\right) A_{Q}-u w^{A} .
\end{aligned}
$$

Note that the $d\left(u_{1}^{\prime}-v_{i}\right)$ contributions vanish because they produce terms like $\Pi_{\cdots} \cdots_{\overline{1}}=0$ (see (28)). We commute the $R_{i j}\left(v_{i j}\right)$ (for $j<i$ ) with $\Pi$ using $(24)$ and apply the $\stackrel{\circ}{R}_{i j}\left(v_{i j}\right)$ to $L$ using (41). Using further Yang-Baxter relations to the $R_{i j}\left(v_{i j}\right)$ (for $j>i$ ) the unwanted terms write as

$$
\begin{aligned}
u w^{A} & =-\sum_{i=1}^{m} c\left(u_{1}^{\prime}-v_{i}\right)\left(L\left(v_{i}, \underline{v}_{i}\right) \Pi\left(v_{i}, \underline{v}_{i}\right)\right)_{\gamma \underline{\beta}_{i}} \\
& \times \prod_{k=1, k \neq i}^{m} a\left(v_{i k}\right) a_{1}\left(\underline{u}, v_{i}\right) \Omega\left[T_{1}^{\beta_{m}}\left(v_{m}\right) \cdots T_{1}^{\beta_{1}}\left(v_{1}\right)\right]_{i}\left(T_{Q}\right)_{1}^{\gamma}
\end{aligned}
$$

where we use the short notations $\underline{v}_{i}, \underline{\beta}_{i}$ and $\left[T_{1}^{\beta_{m}}\left(v_{m}\right) \cdots T_{1}^{\beta_{1}}\left(v_{1}\right)\right]_{i}$ which means that $v_{i}, \beta_{i}$ and $T_{1}^{\beta_{i}}\left(v_{i}\right)$ are missing, respectively. For $\gamma=1$ this vanishes because of $\Pi_{1 \ldots}=0$ (see $(26)$ ), for $\gamma=\dot{\gamma} \neq 1, \overline{1}$ this gives $u w_{C}^{A}$ and for $\gamma=\overline{1}$ this gives $u w_{C_{2}}^{A}$ in the form of (B.7) and (B.10) where (27) and (30) are used.

\section{Proof of Theorem 4.1}

\section{Proof.}

1. The weights $(70)$ of the reference state $\Omega(44)$ are

$$
w=\left(n=n_{0}, 0, \ldots, 0\right)
$$

In level $k=1, \ldots,[(N-3) / 2]$ of the Bethe Ansatz the weights are changed as

$$
w_{k} \rightarrow w_{k}-n_{k}, w_{k+1} \rightarrow w_{k+1}+n_{k} .
$$


This means the states $\Phi_{\underline{\alpha}}(\underline{\dot{\beta}}, \underline{v})$ of 43 are eigenvectors of the weights. Using in addition (56) for $O(3)$ and (64) for $O(4)$ we obtain $w=$

$$
\left(w_{1}, \ldots, w_{[N / 2]}\right)= \begin{cases}\left(n-n_{1}, \ldots, n_{[N / 2]-1}-n_{[N / 2]}, n_{[N / 2]}\right) & \text { for } \quad N \text { odd } \\ \left(n-n_{1}, \ldots, n_{[N / 2]-2}-n_{-}-n_{+}, n_{-}-n_{+}\right) & \text {for } \quad N \text { even. }\end{cases}
$$

2. The proof of the highest weight property

$$
\Psi(\underline{v}) M_{\grave{\gamma}}^{1}=\Psi(\underline{v}) M_{\overline{1}}^{\stackrel{\gamma}{\gamma}}=\Psi(\underline{v}) M_{\overline{1}}^{1}=0
$$

uses similar techniques as the derivation of the unwanted terms.

i) We consider

$$
\begin{aligned}
0 & =(L(\underline{v}) \Pi(\underline{v}))_{\underline{\beta}} \Omega B_{\dot{\gamma}}(v) T_{1}^{\beta_{m}^{\prime}}\left(v_{m}\right) \cdots T_{1}^{\beta_{1}^{\prime}}\left(v_{1}\right) \\
& =(L(\underline{v}) \Pi(\underline{v}))_{\underline{\beta}} \Omega\left(R_{0 m}\left(v-v_{m}\right) \ldots R_{01}\left(v-v_{1}\right)\right)_{\gamma, \underline{\beta}^{\prime}}^{\underline{\beta}, 1} T_{1}^{\beta_{m}^{\prime \prime}}\left(v_{m}\right) \cdots T_{1}^{\beta_{1}^{\prime \prime}}\left(v_{1}\right) T_{\gamma^{\prime}}^{\gamma}(v) \\
& \times\left(R_{01}\left(v_{1}-v\right) \ldots R_{0 m}\left(v_{m}-v\right)\right)_{1 \ldots 1, \gamma}^{\gamma \underline{\beta}^{\prime \prime}}+O\left(v^{-2}\right) .
\end{aligned}
$$

For $v \rightarrow \infty$ applying lemma 2.2, equations (65) and (67) we obtain

$$
0=\Psi(\underline{v}) M_{\dot{\gamma}}^{1}-\sum_{i=1}^{m} X_{\dot{\gamma}}^{(i)}(\underline{u}, \underline{v})+\sum_{i=1}^{m} X_{\dot{\gamma}}^{(i)}\left(\underline{u}, \underline{v}^{(i)}\right) \chi_{i}(\underline{u}, \underline{v})
$$

with $X_{\dot{\gamma}}^{(i)}$ and $\chi_{i}$ defined in B.8) and B.9. After multiplication with $g(\underline{u}, \underline{v})$ and summation over the $\underline{v}$ the terms cancel each other because of $\chi_{i}(\underline{u}, \underline{v}) g(\underline{u}, \underline{v})=$ $g\left(\underline{u}, \underline{v}^{(i)}\right)$.

ii) We consider

$$
\begin{aligned}
& (L(\underline{v}) \Pi(\underline{v}))_{\underline{\beta}}\left(R_{10}\left(v_{1}-v\right) \ldots R_{m 0}\left(v_{m}-v\right)\right)_{\underline{\beta}^{\prime}, \gamma}^{\frac{\gamma}{\beta}} \Omega T_{\overline{1}}^{\gamma}(v) T_{1}^{\beta_{m}^{\prime}}\left(v_{m}\right) \cdots T_{1}^{\beta_{1}^{\prime}}\left(v_{1}\right) \\
& =(L(\underline{v}) \Pi(\underline{v}))_{\underline{\beta}}\left(R_{10}\left(v_{1}-v\right) \ldots R_{m 0}\left(v_{m}-v\right)\right)_{\overline{1}, \underline{\beta}^{\prime}}^{\underline{\gamma}} \Omega T_{1}^{\beta_{m}^{\prime}}\left(v_{m}\right) \cdots T_{1}^{\beta_{1}^{\prime}}\left(v_{1}\right)+O\left(v^{-2}\right) \\
& =\Psi(\underline{v}) T_{\overline{1}}^{\gamma}(v)+O\left(v^{-2}\right) \\
& +(L(\underline{v}) \Pi(\underline{v}))_{\underline{\beta}} \Omega T_{\beta_{m}^{\prime}}^{\beta_{m}}\left(v_{m}\right) \cdots T_{\beta_{1}^{\prime}}^{\beta_{1}}\left(v_{1}\right)\left(R_{10}\left(v_{1}-v\right) \ldots R_{m 0}\left(v_{m}-v\right)\right)_{1 \ldots 1, \overline{1}}^{\frac{\gamma}{\beta^{\prime}}} .
\end{aligned}
$$

It has been used that only $\gamma=\overline{1}$ contributes because of $\Omega B_{2}=\Omega B_{3}=0$.

For $v \rightarrow \infty$ applying lemma 2.2, equations (65) and (67) we obtain

$$
0=\Psi(\underline{v}) M_{\overline{1}}^{\dot{\gamma}}-\mathbf{C}^{\dot{\gamma} \hat{\gamma}^{\prime}} \sum_{i=1}^{m} X_{\dot{\gamma}^{\prime}}^{(i)}(\underline{u}, \underline{v})+\mathbf{C}^{\gamma^{\gamma} \hat{\gamma}^{\prime}} \sum_{i=1}^{m} X_{\hat{\gamma}^{\prime}}^{(i)}\left(\underline{u}, \underline{v}^{(i)}\right) \chi_{i}(\underline{u}, \underline{v}) .
$$

Again after multiplication with $g(\underline{u}, \underline{v})$ and summation over the $\underline{v}$ the terms cancel each other because of $\chi_{i}(\underline{u}, \underline{v}) g(\underline{u}, \underline{v})=g\left(\underline{u}, \underline{v}^{(i)}\right)$. 
iii) We consider

$$
\begin{aligned}
0 & =\Omega M_{\overline{1}}^{1} \ldots \\
& =(L(\underline{v}) \Pi(\underline{v}))_{\underline{\beta}}\left(R\left(v-v_{m}\right) \ldots R\left(v-v_{1}\right)\right)_{\gamma, \underline{\beta^{\prime}}}^{\beta, 1} \Omega T_{\beta_{m}^{\prime \prime}}^{\beta_{m}^{\prime}}\left(v_{m}\right) \cdots T_{\beta_{1}^{\prime \prime}}^{\beta_{1}^{\prime}}\left(v_{1}\right) T_{\gamma^{\prime}}^{\gamma}(v) \\
& \times\left(R\left(v_{1}-v\right) \ldots R\left(v_{m}-v\right)\right)_{1, \ldots 1, \overline{1}}^{\gamma^{\prime}, \beta^{\prime \prime}} .
\end{aligned}
$$

For $v \rightarrow \infty$ we apply lemma 2.2 , equations (65) and (67) and obtain

$$
0=\Psi(\underline{v}) M_{\overline{1}}^{1}-\sum_{i=1}^{m} X^{(i j)}(\underline{v})+\sum_{i=1}^{m} X^{(i j)}\left(v_{i}^{\prime}, v_{j}^{\prime}, \underline{v}_{i j}\right) \chi_{i}(\underline{u}, \underline{v}) \chi_{j}(\underline{u}, \underline{v}) .
$$

Again after multiplication with $g(\underline{u}, \underline{v})$ and summation over the $\underline{v}$ the terms cancel each other because of $\chi_{i}(\underline{u}, \underline{v}) \chi_{j}(\underline{u}, \underline{v}) g(\underline{u}, \underline{v})=g\left(\underline{u}, \underline{v}^{(i j)}\right)$.

iv) Next we prove

$$
\Psi(\underline{w}) M_{\dot{\gamma}}^{\dot{\gamma}^{\prime}}=0,1<\dot{\gamma}^{\prime}<\dot{\gamma}<\overline{1}
$$

We consider

$$
\begin{aligned}
& L_{\underline{\beta^{\prime}}}(\underline{w}) \Pi_{\gamma \underline{\gamma^{\prime}}}^{\hat{\gamma}^{\prime}}(v, \underline{w}) \Omega T_{1}^{\beta_{m}}\left(w_{m}\right) \cdots T_{1}^{\beta_{1}}\left(w_{1}\right) T_{\dot{\gamma}}^{\gamma}(v)+O\left(v^{-2}\right) \\
& =\left(L(\underline{w})\left(T^{(1)}\right)_{\dot{\gamma}}^{\dot{\gamma}^{\prime}}(v)\right)_{\underline{\beta}^{\prime}} \Pi_{\underline{\beta}}^{\underline{\beta}^{\prime}}(\underline{w}) \Omega T_{\dot{\gamma}}^{\gamma}(v) T_{1}^{\beta_{m}}\left(w_{m}\right) \cdots T_{1}^{\beta_{1}}\left(w_{1}\right)+O\left(v^{-2}\right)
\end{aligned}
$$

where Yang-Baxter rules and (24) have been used. We have also used that by (30) and (15)

$$
\begin{aligned}
\Pi_{\gamma \underline{\underline{\beta}}}^{\hat{\gamma}^{\prime}} & \underline{\underline{\beta}}^{\prime}(v, \underline{w})=\delta_{\gamma}^{\gamma^{\prime}} \Pi_{\underline{\beta}}^{\underline{\beta}^{\prime}}(\underline{w})+O\left(v^{-1}\right) \\
\left(R\left(w_{1}-v\right) \ldots R\left(w_{m}-v\right)\right)_{1 \ldots m, 0} & =\mathbf{1}_{1 \ldots m} \mathbf{1}_{0}+O\left(v^{-1}\right) .
\end{aligned}
$$

For $v \rightarrow \infty$ the highest weight condition $L(\underline{w})\left(M^{(1)}\right)_{\dot{\gamma}}^{\dot{\gamma}^{\prime}}=0$ implies the claim.

3. The highest weight properties of the weights are obtained as follows. In the complex basis the relation (74) reads as

$$
\left[M_{\alpha}^{\alpha^{\prime}}, M_{\beta}^{\beta^{\prime}}\right]=-\delta_{\alpha}^{\beta^{\prime}} M_{\beta}^{\alpha^{\prime}}+\mathbf{C}^{\alpha^{\prime} \beta^{\prime}}(\mathbf{C} M)_{\alpha \beta}+M_{\alpha}^{\beta^{\prime}} \delta_{\beta}^{\alpha^{\prime}}-(M \mathbf{C})^{\beta^{\prime} \alpha^{\prime}} \mathbf{C}_{\alpha \beta}
$$

In particular for $\beta \neq \alpha, \bar{\alpha}$

$$
\left[M_{\alpha}^{\beta}, M_{\beta}^{\alpha}\right]=M_{\alpha}^{\alpha}-M_{\beta}^{\beta}=M_{\alpha}^{\alpha}+M_{\bar{\beta}}^{\bar{\beta}} .
$$

Because of $\left(M_{\alpha}^{\beta}\right)^{\dagger}=M_{\beta}^{\alpha}$

$$
0 \leq M_{\alpha}^{\beta}\left(M_{\alpha}^{\beta}\right)^{\dagger}=M_{\alpha}^{\beta} M_{\beta}^{\alpha}=M_{\beta}^{\alpha} M_{\alpha}^{\beta}+M_{\alpha}^{\alpha}-M_{\beta}^{\beta} .
$$


For highest weight co-vectors

$$
0=\Psi M_{\beta}^{\alpha} \text { for } \alpha<\beta
$$

which implies for the weights $(70)$

$$
0 \leq w_{\alpha}-w_{\beta} \text { for } \alpha<\beta \leq N / 2 \text {. }
$$

In addition if $N$ is even

$$
\begin{aligned}
0 & \leq w_{\alpha}+w_{\bar{\beta}} \text { for } \alpha \leq N / 2<\beta \neq \bar{\alpha} \\
& \Rightarrow w_{1} \geq w_{2} \geq \cdots \geq w_{N / 2-1} \geq\left|w_{N / 2}\right|
\end{aligned}
$$

and if $N$ is odd

$$
\begin{aligned}
0 & \leq w_{\alpha} \text { for } \alpha \leq N / 2 \text { because } \Psi M_{0}^{0}=0 \\
& \Rightarrow w_{1} \geq w_{2} \geq \cdots \geq w_{N / 2} \geq 0
\end{aligned}
$$

\section{References}

[1] M. Karowski and P. Weisz, Exact form factors in $(1+1)$-dimensional field theoretic models with soliton behavior, Nucl. Phys. B139, 455-476 (1978).

[2] B. Berg, M. Karowski, and P. Weisz, Construction of Green functions from an exact S matrix, Phys. Rev. D19, 2477-2479 (1979).

[3] F. Smirnov, Form Factors in Completely Integrable Models of Quantum Field Theory, Adv. Series in Math. Phys. 14, World Scientific (1992).

[4] H. Babujian, M. Karowski, and A. Zapletal, Matrix Difference Equations and a Nested Bethe Ansatz, J. Phys. A30, 6425-6450 (1997).

[5] H. M. Babujian, A. Fring, M. Karowski, and A. Zapletal, Exact form factors in integrable quantum field theories: The sine-Gordon model, Nucl. Phys. B538, 535586 (1999).

[6] H. M. Babujian, A. Foerster, and M. Karowski, The nested SU(N) off-shell Bethe ansatz and exact form factors, J. Phys. A41, 275202 (2008).

[7] H. Bethe, On the theory of metals. 1. Eigenvalues and eigenfunctions for the linear atomic chain, Z. Phys. 71, 205-226 (1931).

[8] L. A. Takhtajan and L. D. Faddeev, The Quantum method of the inverse problem and the Heisenberg XYZ model, Russ. Math. Surveys 34, 11-68 (1979). 
[9] H. M. Babujian, Off-shell Bethe Ansatz equation and N point correlators in SU(2) WZNW theory, J. Phys. A26, 6981-6990 (1993).

[10] N. Y. Reshetikhin, Jackson-type integrals, Bethe vectors, and solutions to a difference analog of the Knizhnik-Zamolodchikov system, Lett. Math. Phys. 26, 153-165 (1992).

[11] C. N. Yang, Some exact results for the many body problems in one dimension with repulsive delta function interaction, Phys. Rev. Lett. 19, 1312-1314 (1967).

[12] B. Sutherland, Further Results for the Many-Body Problem in One Dimension, Phys.Rev.Lett. 20, 98-100 (1968).

[13] B. Sutherland, Model for a multicomponent quantum system, Phys. Rev. B 12(9), 3795-3805 (Nov 1975).

[14] H. Babujian, M. Karowski, and A. Zapletal, SU(N) Matrix Difference Equations and a Nested Bethe Ansatz, hep-th/9611005 (1996).

[15] V. Tarasov, Algebraic Bethe Ansatz for the Izergin-Korepin R Matrix, Theor. Math. Phys. 76, 793-803 (1988).

[16] M. J. Martins and P. B. Ramos, The algebraic Bethe ansatz for rational braid-monoid lattice models, Nucl. Phys. B500, 579-620 (1997).

[17] H. J. de Vega and M. Karowski, Exact Bethe ansatz solution of $\mathrm{O}(2 \mathrm{~N})$ symmetric theories, Nucl. Phys. B280, 225 (1987).

[18] H. M. Babujian, A. Foerster, and M. Karowski, Exact form factors of the $\mathrm{O}(\mathrm{N})$ Sigma and Gross-Neveu models and the 1/N expansion, to be published.

[19] J. Balog, Form-factors and asymptotic freedom in the $\mathrm{O}(3)$ sigma model, Phys. Lett. B300, 145-151 (1993).

[20] J. Balog and T. Hauer, Polynomial form-factors in the $\mathrm{O}(3)$ nonlinear sigma model, Phys. Lett. B337, 115-121 (1994).

[21] L. F. Alday, D. Gaiotto, and Y. Tachikawa, Liouville Correlation Functions from Four-dimensional Gauge Theories, Lett. Math. Phys. 91, 167-197 (2010).

[22] A. Mironov, A. Morozov, S. Shakirov, and A. Smirnov, Proving AGT conjecture as HS duality: extension to five dimensions, (2011), arXiv:1105.0948 [hep-th].

[23] I. B. Frenkel and N. Y. Reshetikhin, Quantum affine algebras and holonomic difference equations, Commun. Math. Phys. 146, 1-60 (1992).

[24] A. B. Zamolodchikov and A. B. Zamolodchikov, Factorized S-matrices in two dimensions as the exact solutions of certain relativistic quantum field models, Annals Phys. 120, 253-291 (1979). 
[25] L. D. Faddeev, E. K. Sklyanin, and L. A. Takhtajan, The quantum inverse problem method. 1, Theor. Math. Phys. 40, 688-706 (1980).

[26] H. Babujian, A. Foerster, and M. Karowski, Exact form factors in integrable quantum field theories: The scaling Z(N)-Ising model, Nucl. Phys. B736, 169-198 (2006).

[27] Y. Takeyama, Form factors of SU(N) invariant Thirring model, Publ. Res. Inst. Math. Sci. Kyoto 39, 59-116 (2003).

[28] R. Shankar and E. Witten, The S matrix of the kinks of the (psi-bar PSI)**2 model, Nucl. Phys. B141, 349 (1978).

[29] M. Karowski and H. J. Thun, Complete S matrix of the $\mathrm{O}(2 \mathrm{~N})$ Gross-Neveu model, Nucl. Phys. B190, 61-92 (1981).

[30] H. Babujian, A. Foerster, and M. Karowski, Exact form factors of the SU(N) GrossNeveu model and 1/N expansion, Nucl. Phys. B825, 396-425 (2010).

[31] H. M. Babujian, A. Foerster, and M. Karowski, The Nested Off-shell Bethe Ansatz and $\mathrm{O}(\mathrm{N})$ Matrix Difference Equations, to be published. 